\title{
Extracting the Frequency-Dependent Dynamic Stokes Shift from Two-Dimensional Electronic Spectra with Prominent Vibrational Coherences
}

\author{
Jiawei Lu, Yumin Lee, Jessica M. Anna* \\ University of Pennsylvania, 231 South 34 Street, Philadelphia, Pennsylvania 19104, United States
}

\section{Supporting Information}

Table of Contents

\begin{tabular}{|c|c|c|}
\hline 1. & Pulse Characterization... & $\mathrm{S} 2$ \\
\hline 2. & DFT and TD-DFT Calculations.................................................... & $\mathrm{S} 3$ \\
\hline 3. & $\begin{array}{l}\text { Procedure to Extract the Timescale of the Inertial Response from the } 2 \text { DES } \\
\text { Spectra } \ldots \ldots \ldots \ldots \ldots \ldots \ldots \ldots \ldots \ldots \ldots \ldots \ldots \ldots \ldots \ldots \ldots \ldots \ldots \ldots \ldots \ldots \ldots \ldots \ldots \ldots \ldots \ldots \ldots \ldots \ldots \ldots \ldots \ldots\end{array}$ & S9 \\
\hline 4. & 2DES of Cresyl Violet Obtained Under Different Pump Pulse Tunings.............. & S15 \\
\hline 5. & Excitation Frequency Dependence of Spectral Diffusion from Model Spectra............ & S21 \\
\hline 6. & Comparison of a Global Analysis and the Dynamic Stokes Shift.... & S24 \\
\hline 7. & References...... & S27 \\
\hline
\end{tabular}




\section{Pulse Characterization}

The incoming pulses were characterized by sum frequency generation frequency resolved optical gating (SFG-FROG) ${ }^{1}$ using a $100 \mu \mathrm{m} \mathrm{BBO}$ crystal as the nonlinear medium. The cross-correlation trace for the pulses used in the 2DES measurements presented in the main text is shown in Figure $\mathrm{S} 1$. The cross-correlation has a temporal FWHM of 21 fs from the projection of the SFG-FROG onto the time axis.

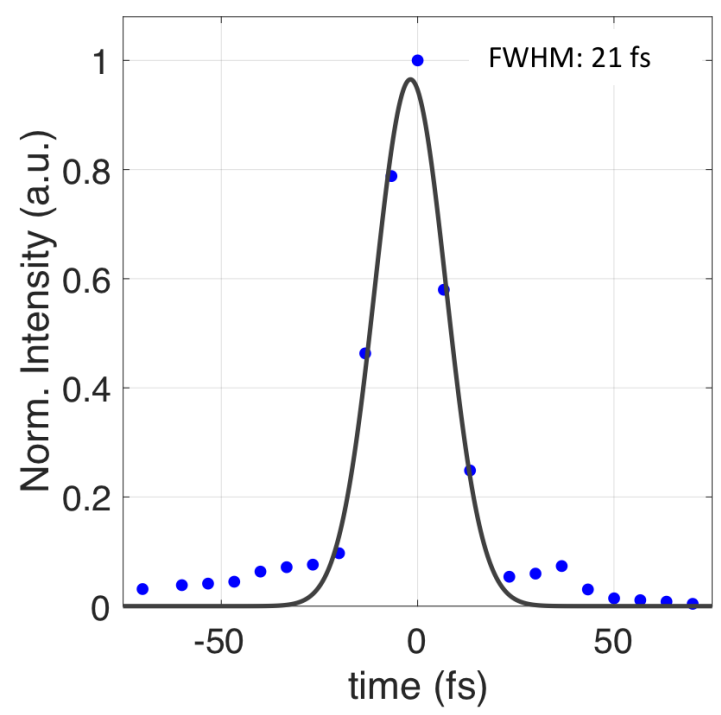

Figure S1. Intensity of the projection of the SFG-FROG onto the time axis. 


\section{DFT and TD-DFT Calculations}

DFT and TD-DFT calculations were performed at the B3LYP level of theory using the 6$311+\mathrm{G}(2 \mathrm{~d}, \mathrm{p})$ basis set with the Gaussian 16 software package. ${ }^{2}$ The calculations employed the Polarizable Continuum Model (PCM) to account for the methanol solvent.

\subsection{Optimized Ground State Geometry of Cresyl Violet}

The DFT optimized ground state structure of cresyl violet is shown in Figure S2 and the cartesian coordinates are reported in Table S1.

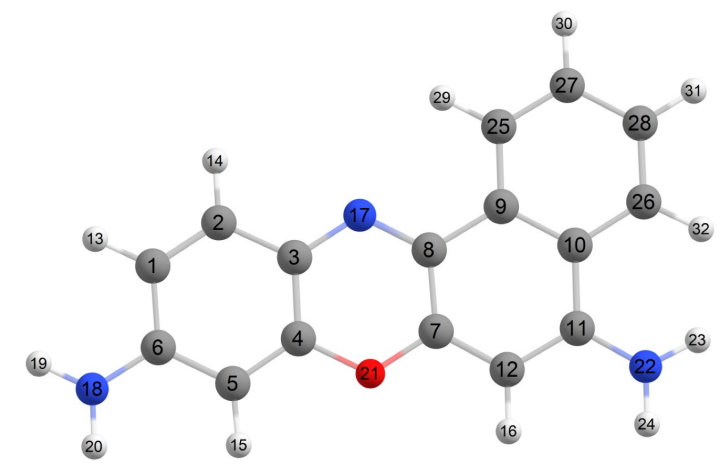

Figure S2. The optimized ground state structure of cresyl violet.

Table S1. The Cartesian coordinates for the DFT optimized ground state of cresyl violet.

\begin{tabular}{|c|c|c|c|c|c|}
\hline \multirow{2}{*}{$\begin{array}{l}\text { Center } \\
\text { Number }\end{array}$} & \multirow{2}{*}{$\begin{array}{l}\text { Atomic } \\
\text { Number }\end{array}$} & \multirow{2}{*}{$\begin{array}{l}\text { Atomic } \\
\text { Type }\end{array}$} & \multicolumn{3}{|c|}{ Coordinates (Angstroms) } \\
\hline & & & $x$ & $\mathrm{Y}$ & Z \\
\hline 1 & 6 & 0 & 4.140428 & -1.345600 & 0.000743 \\
\hline 2 & 6 & 0 & 2.842016 & -1.759471 & 0.000619 \\
\hline 3 & 6 & 0 & 1.779937 & -0.819102 & 0.000359 \\
\hline 4 & 6 & 0 & 2.129107 & 0.559598 & 0.000237 \\
\hline 5 & 6 & 0 & 3.428424 & 0.999793 & 0.000361 \\
\hline 6 & 6 & 0 & 4.465102 & 0.046624 & 0.000616 \\
\hline 7 & 6 & 0 & -0.159730 & 1.086552 & -0.000156 \\
\hline 8 & 6 & 0 & -0.459886 & -0.309970 & -0.000027 \\
\hline 9 & 6 & 0 & -1.860254 & -0.697565 & -0.000179 \\
\hline 10 & 6 & 0 & -2.869364 & 0.296822 & -0.000435 \\
\hline 11 & 6 & 0 & -2.481878 & 1.702011 & -0.000558 \\
\hline 12 & 6 & 0 & -1.126048 & 2.060950 & -0.000412 \\
\hline 13 & 1 & 0 & 4.945877 & -2.069143 & 0.000938 \\
\hline 14 & 1 & 0 & 2.589569 & -2.812043 & 0.000710 \\
\hline 15 & 1 & 0 & 3. 644419 & 2.060406 & 0.000263 \\
\hline 16 & 1 & 0 & -0.835549 & 3. 103386 & -0.000493 \\
\hline 17 & 7 & 0 & 0.499256 & -1.219506 & 0.000225 \\
\hline 18 & 7 & 0 & 5.754754 & 0.430807 & 0.000740 \\
\hline 19 & 1 & 0 & 6.499909 & -0.246727 & 0.000914 \\
\hline 20 & 1 & 0 & 6.011786 & 1.404581 & 0.000657 \\
\hline 21 & 8 & 0 & 1.130952 & 1.484121 & -0.000017 \\
\hline 22 & 7 & 0 & -3.406891 & 2.665643 & -0.000805 \\
\hline 23 & 1 & 0 & -4.393205 & 2.469185 & -0.000936 \\
\hline 24 & 1 & 0 & -3.135966 & 3.636831 & -0.000901 \\
\hline 25 & 6 & 0 & -2.227951 & -2.051901 & -0.000074 \\
\hline 26 & 6 & 0 & -4.217713 & -0.104286 & -0.000563 \\
\hline 27 & 6 & 0 & -3.558099 & -2.419970 & -0.000212 \\
\hline 28 & 6 & 0 & -4.558900 & -1.441549 & -0.000454 \\
\hline 29 & 1 & 0 & -1.448755 & -2.801299 & 0.000116 \\
\hline 30 & 1 & 0 & -3.827545 & -3.468800 & -0.000132 \\
\hline 31 & 1 & 0 & -5.602064 & -1.730473 & -0.000557 \\
\hline 32 & 1 & 0 & -5.017063 & 0.624890 & -0.000747 \\
\hline
\end{tabular}




\subsection{Optimized Excited State Geometry for Cresyl Violet}

To obtain the optimized geometry of the $\mathrm{S}_{1}$ state, the vertical transitions were first calculated at the optimized ground state geometry for the first 10 singlet excited states using the keyword TD(NStates $=10)$. This was followed by TD-DFT calculations to obtain the optimized geometry of the first singlet excited electronic state. The TD-DFT optimized geometry of the $\mathrm{S}_{1}$ state is shown in Figure S3 and the Cartesian coordinates are reported in Table S2.

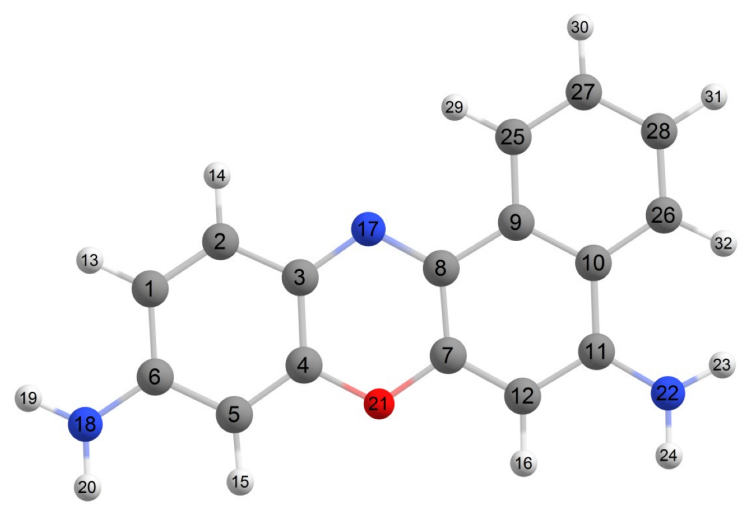

Figure S3. The optimized structure of the $\mathrm{S}_{1}$ excited electronic state.

Table S2. The Cartesian coordinates for the TD-DFT optimized $\mathrm{S}_{1}$ excited state of cresyl violet.

\begin{tabular}{|c|c|c|c|c|c|}
\hline \multirow{2}{*}{$\begin{array}{l}\text { Center } \\
\text { Number }\end{array}$} & \multirow{2}{*}{$\begin{array}{l}\text { Atomic } \\
\text { Number }\end{array}$} & \multirow{2}{*}{$\begin{array}{l}\text { Atomic } \\
\text { Type }\end{array}$} & \multicolumn{3}{|c|}{ Coordinates (Angstroms) } \\
\hline & & & $x$ & $\mathrm{Y}$ & Z \\
\hline 1 & 6 & 0 & 4.158018 & -1.342612 & 0.000751 \\
\hline 2 & 6 & 0 & 2.854273 & -1.755100 & 0.000606 \\
\hline 3 & 6 & 0 & 1.778875 & -0.826013 & 0.000336 \\
\hline 4 & 6 & 0 & 2.119819 & 0.554042 & 0.000211 \\
\hline 5 & 6 & 0 & 3.419232 & 0.995702 & 0.000345 \\
\hline 6 & 6 & 0 & 4.471951 & 0.048621 & 0.000629 \\
\hline 7 & 6 & 0 & -0.164272 & 1.074420 & -0.000175 \\
\hline 8 & 6 & 0 & -0.461708 & -0.316850 & -0.000044 \\
\hline 9 & 6 & 0 & -1.854358 & -0.686661 & -0.000184 \\
\hline 10 & 6 & 0 & -2.875542 & 0.307949 & -0.000430 \\
\hline 11 & 6 & 0 & -2.498949 & 1.705172 & -0.000557 \\
\hline 12 & 6 & 0 & -1.134550 & 2.049590 & -0.000421 \\
\hline 13 & 1 & 0 & 4.963479 & -2.066151 & 0.000960 \\
\hline 14 & 1 & 0 & 2.606755 & -2.809053 & 0.000696 \\
\hline 15 & 1 & 0 & 3.629422 & 2.057796 & 0.000241 \\
\hline 16 & 1 & 0 & -0.836533 & 3.090619 & -0.000498 \\
\hline 17 & 7 & 0 & 0.493578 & -1.268701 & 0.000206 \\
\hline 18 & 7 & 0 & 5.753348 & 0.457690 & 0.000783 \\
\hline 19 & 1 & 0 & 6.513723 & -0.205424 & 0.000972 \\
\hline 20 & 1 & 0 & 5.992649 & 1.437705 & 0.000726 \\
\hline 21 & 8 & 0 & 1.133182 & 1.501365 & -0.000051 \\
\hline 22 & 7 & 0 & -3.417600 & 2.685947 & -0.000794 \\
\hline 23 & 1 & 0 & -4.406699 & 2.502472 & -0.000927 \\
\hline 24 & 1 & 0 & -3.134145 & 3.653491 & -0.000892 \\
\hline 25 & 6 & 0 & -2.219045 & -2.046425 & -0.000076 \\
\hline 26 & 6 & 0 & -4.216067 & -0.109771 & -0.000536 \\
\hline 27 & 6 & 0 & -3.549539 & -2.428339 & -0.000198 \\
\hline 28 & 6 & 0 & -4.551382 & -1.456261 & -0.000425 \\
\hline 29 & 1 & 0 & -1.432957 & -2.788389 & 0.000104 \\
\hline 30 & 1 & 0 & -3.810327 & -3.479048 & -0.000116 \\
\hline 31 & 1 & 0 & -5.594200 & -1.746503 & -0.000514 \\
\hline 32 & 1 & 0 & -5.022441 & 0.612224 & -0.000707 \\
\hline
\end{tabular}




\subsection{Ground State Frequency Calculation}

The non-resonant Raman spectrum of cresyl violet was calculated from the optimized ground state geometry (Figure S2, Table S1) using the Freq=Raman keyword. Figure S4 plots the calculated Raman activity in the $300 \mathrm{~cm}^{-1}$ to $800 \mathrm{~cm}^{-1}$ spectral region (cyan bars) along with the experimentally extracted power spectrum obtained from the Fourier transform of the $t_{2}$ dependent residuals as described in the main text (blue line). The experimental frequencies are indicated as dashed vertical blue lines at 343, 470,520,567, 587 and $666 \mathrm{~cm}^{-1}$. The experimental transitions are assigned by comparing the intensities and frequencies to those of the calculated spectrum. We note that a scaling factor was not applied to the calculated frequencies. The assignment follows: $343 \mathrm{~cm}^{-1}$ is assigned to $v_{13}=347 \mathrm{~cm}^{-1} ; 470 \mathrm{~cm}^{-1}$ is assigned to $v_{19}=472 \mathrm{~cm}^{-1} ; 520 \mathrm{~cm}^{-1}$ is assigned to $v_{23}=531 \mathrm{~cm}^{-1} ; 587 \mathrm{~cm}^{-1}$ is assigned to $v_{26}=608 \mathrm{~cm}^{-1} ; 666 \mathrm{~cm}^{-1}$ is assigned to $v_{30}=687 \mathrm{~cm}^{-1}$. The $567 \mathrm{~cm}^{-1}$ peak is not assigned to a Raman active mode. This mode is assigned to an excited state vibrational coherence in section 2.4 of the Supporting Information, consistent with previous experimental studies. ${ }^{3}$ The nuclear motions associated with the Raman active modes, $v_{13}, v_{19}, v_{23}$, $v_{26}$ and $v_{30}$, are depicted with blue arrows superposed on the optimized ground state geometry in Figure S5.

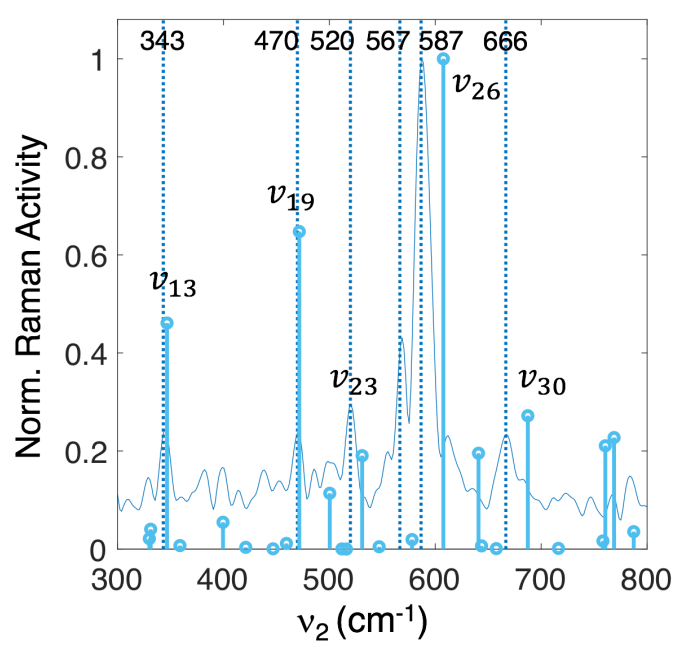

Figure S4. Calculated Raman spectrum of cresyl violet (cyan stick spectra) and experimental power spectrum (blue). The experimental frequencies are indicated by the dashed vertical lines. The assigned vibrational modes are indicated with the mode number obtained from the DFT calculations. 

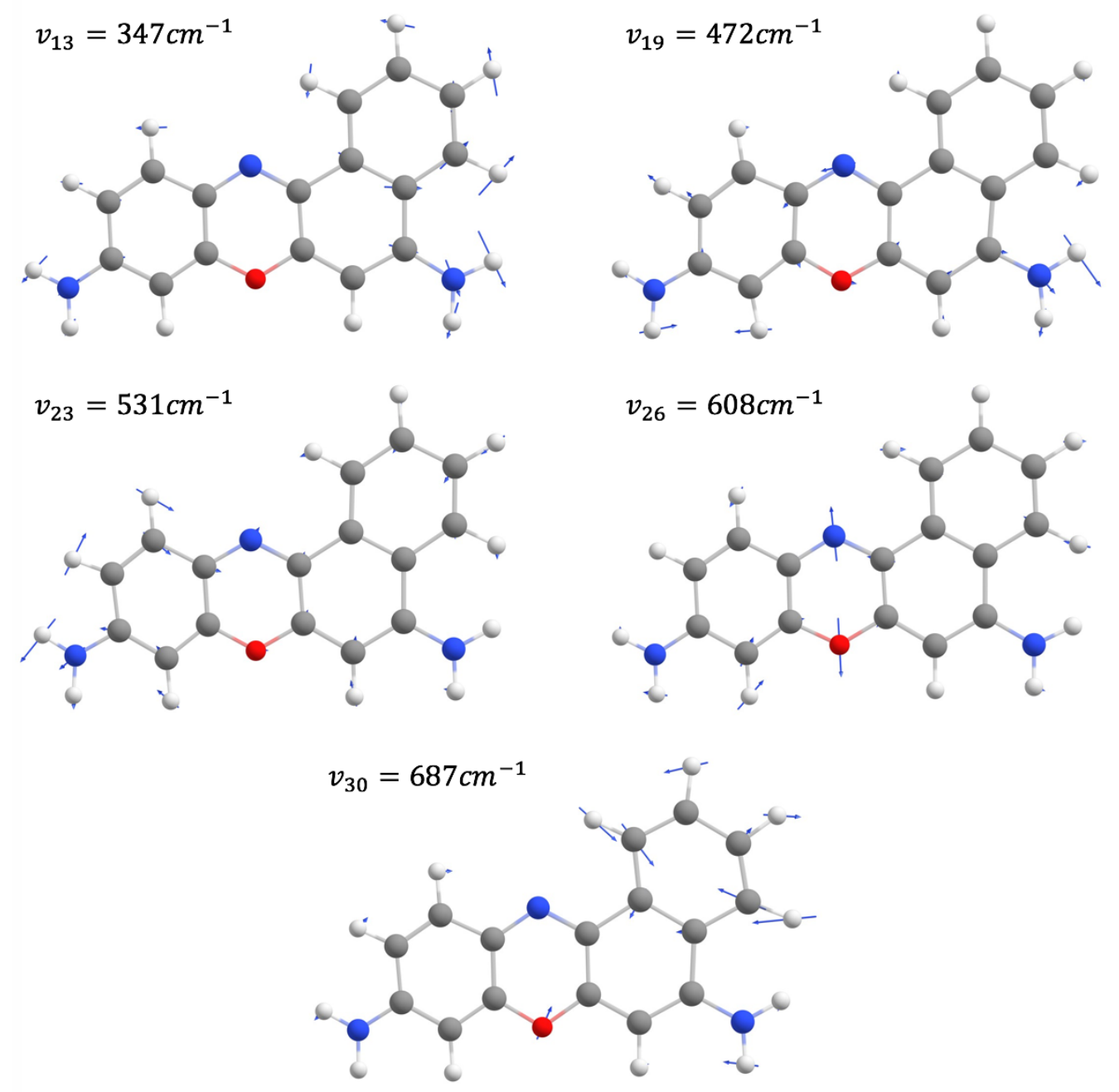

Figure S5. The nuclear motions associated with the Raman active modes are indicated with blue arrows superposed on the optimized ground state geometry. The modes are denoted with mode number and calculated frequency. 


\subsection{Calculation of the Franck-Condon Active Modes for Cresyl Violet}

The Franck-Condon (FC) active modes for absorption and emission were calculated using the Freq(FC, ReadFC) keywords. The frequency calculations of the optimized ground electronic state and first excited electronic state (where we have used the Freq=SaveNormalModes keyword) were used as inputs. We note the excited state and ground state frequency calculations lacked negative frequencies, confirming the optimized geometries are at minima.

The calculated FC dipole strengths for absorption (FC abs., magenta bars in Fig S6) and emission (FC em., orange bars in Fig S6) are plotted as a function of wavenumber in the 300 to $800 \mathrm{~cm}^{-1}$ spectral region in Figure S6. For comparison, the FC dipole strengths for both emission and absorption were normalized to the maximum intensity of the $\mathrm{FC}$ emission factor at $608 \mathrm{~cm}^{-1}$. The FC dipole strengths are plotted along with the experimental power spectrum in blue with the frequencies of the experimental coherences indicated by dashed vertical blue lines. The experimental coherences are assigned to the FC active modes through comparison of the calculated and experimentally determined frequencies. The assignment follows: the experimental $343 \mathrm{~cm}^{-1}$ mode is assigned to $\mathrm{FC}_{\mathrm{abs}}=342 \mathrm{~cm}^{-1}$ and $\mathrm{FC}_{\mathrm{em}}=347 \mathrm{~cm}^{-1}$; the experimental $470 \mathrm{~cm}^{-1}$ mode is assigned to $\mathrm{FC}_{\mathrm{em}}=472 \mathrm{~cm}^{-1}$; the experimental $520 \mathrm{~cm}^{-1}$ mode is assigned to $\mathrm{FC}_{\mathrm{abs}}=533 \mathrm{~cm}^{-1}$ and $\mathrm{FC}_{\mathrm{em}}=531 \mathrm{~cm}^{-1}$; the experimental $567 \mathrm{~cm}^{-1}$ mode is assigned to $\mathrm{FC}_{\mathrm{abs}}=572 \mathrm{~cm}^{-1}$; the experimental $587 \mathrm{~cm}^{-1}$ mode is assigned to $\mathrm{FC}_{\mathrm{abs}}=597$ and $\mathrm{FC}_{\mathrm{em}}=608 \mathrm{~cm}^{-1}$. We note that dual assignments are made where we do not have the experimental resolution to isolate the FC absorption from the FC emission. The initial and final vibrational states associated with the $\mathrm{FC}$ absorption and emission are reported in Table S3. We note that the experimentally observed $666 \mathrm{~cm}^{-1}$ coherence is absent in Franck-Condon calculations. As discussed in previous section (2.3 of the SI) this mode is assigned to a ground state vibrational coherence $\left(v_{30}=687 \mathrm{~cm}^{-1}\right)$ excited through Raman processes.

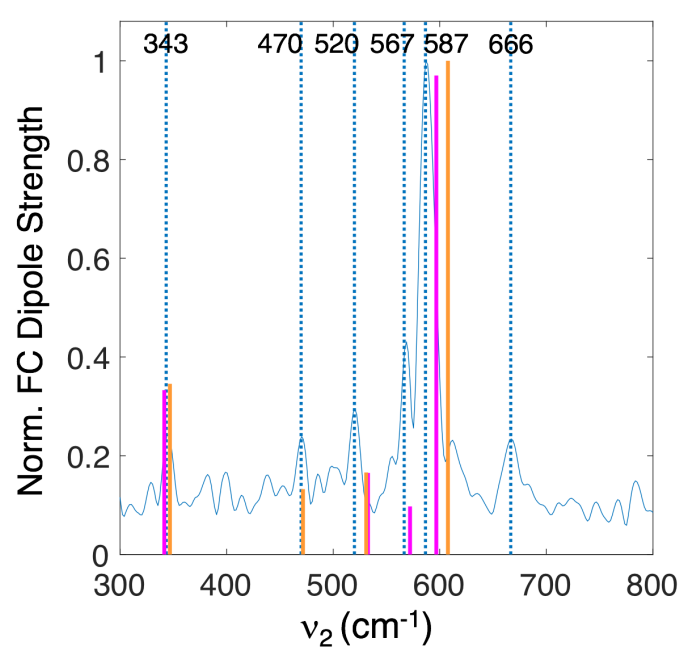

Figure S6. Calculated Franck-Condon dipole strengths are plotted as a function of frequency for the absorption (magenta) and emission (orange). The experimental power spectrum of cresyl violet is shown in blue and the frequencies of the experimental coherences are indicated as dashed blue vertical lines. 
Table S3. Information pertaining to the calculated FC absorption and emission is given. Note that the initial state corresponds to the lowest lying vibrational state $(\mathrm{v}=0)$ in either the ground electronic state for FC absorption or the excited electronic state for FC emission. The 0 frequency FC dipole strength corresponds to the electronic transition from the $\mathrm{v}=0$ states.

\begin{tabular}{|c|c|c|c|c|c|}
\hline \multicolumn{3}{|c|}{ FC Absorption } & \multicolumn{3}{c|}{ FC Emission } \\
\hline $\begin{array}{c}\text { Frequency } \\
\left(\mathbf{c m}^{-1}\right)\end{array}$ & $\begin{array}{c}\text { Final } \\
\text { State }\end{array}$ & $\begin{array}{c}\text { Dipole } \\
\text { Strength (au) }\end{array}$ & $\begin{array}{c}\text { Frequency } \\
\left(\mathbf{c m}^{-1}\right)\end{array}$ & $\begin{array}{c}\text { Final } \\
\text { State }\end{array}$ & $\begin{array}{c}\text { Dipole } \\
\text { Strength (au) }\end{array}$ \\
\hline $\mathbf{0}$ & 0 & 9.784 & $\mathbf{0}$ & 0 & 9.784 \\
\hline $\mathbf{2 5 3}$ & $v_{9}$ & 0.5391 & $\mathbf{- 2 5 9}$ & $v_{9}$ & 0.4946 \\
\hline $\mathbf{3 4 2}$ & $v_{13}$ & 0.7691 & $\mathbf{- 3 4 7}$ & $v_{13}$ & 0.7988 \\
\hline $\mathbf{5 3 3}$ & $v_{24}$ & 0.3817 & $\mathbf{- 4 7 2}$ & $v_{19}$ & 0.3058 \\
\hline $\mathbf{5 7 2}$ & $v_{25}$ & 0.2249 & $\mathbf{- 5 3 1}$ & $v_{23}$ & 0.3838 \\
\hline $\mathbf{5 9 7}$ & $v_{26}$ & 2.242 & $\mathbf{- 6 0 8}$ & $v_{26}$ & 2.311 \\
\hline $\mathbf{8 3 0}$ & $v_{38}$ & 0.2480 & $\mathbf{- 8 4 3}$ & $v_{38}$ & 0.2166 \\
\hline $\mathbf{1 1 9 4}$ & $2 v_{26}$ & 0.2379 & $\mathbf{- 1 2 1 5}$ & $2 v_{26}$ & 0.2949 \\
\hline $\mathbf{1 3 8 1}$ & $v_{65}$ & 0.2693 & $\mathbf{- 1 4 3 4}$ & $v_{65}$ & 0.2536 \\
\hline $\mathbf{1 5 4 3}$ & $v_{72}$ & 0.3326 & & & \\
\hline
\end{tabular}




\section{Procedure to Extract the Timescale of the Inertial Response from the 2DES Spectra}

The general procedure for the extraction of $v_{\mathrm{SE}}\left(\mathrm{t}_{2}\right)$ and $v_{\mathrm{GSB}}\left(\mathrm{t}_{2}\right)$ from the 2DES spectra is described in our previous work ${ }^{4}$ and in the main text. To extract $v_{\mathrm{GSB} / \mathrm{SE}}$, we first determine $v_{\mathrm{MAX}}$, the frequency associated with the maximum amplitude of the projection. $v_{\mathrm{SE}}$ is obtained from taking the frequency closest to $50 \%$ of the maximum amplitude of the projection for $v<v_{\mathrm{MAX}}$ and $v_{\mathrm{GSB}}$ is reporting on the frequency closest to $50 \%$ of the maximum amplitude of the projection for $v>$

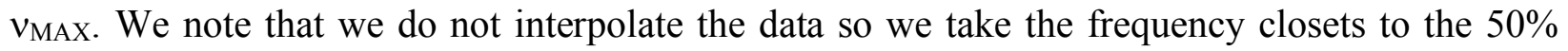
value. Figure $\mathrm{S} 7 \mathrm{c}$ displays $v_{\mathrm{GSB} / \mathrm{SE}}\left(\mathrm{t}_{2}\right)$ extracted from the projection of the 2DES spectra of cresyl violet onto the $v_{3}$ axis. We note that over the course of the first $200 \mathrm{fs} v_{\mathrm{GSB}}$ remains nearly constant while $v_{\mathrm{SE}}$ decreases. As the energy gap associated with the ground state bleach should not change as a function of $t_{2}$ time, we expect the frequency of $v_{\mathrm{GSB}}$ to remain constant at early times. On longer timescales $v_{\mathrm{GSB}}$ begins to evolve and increases as spectral diffusion and vibrational energy transfer take place.

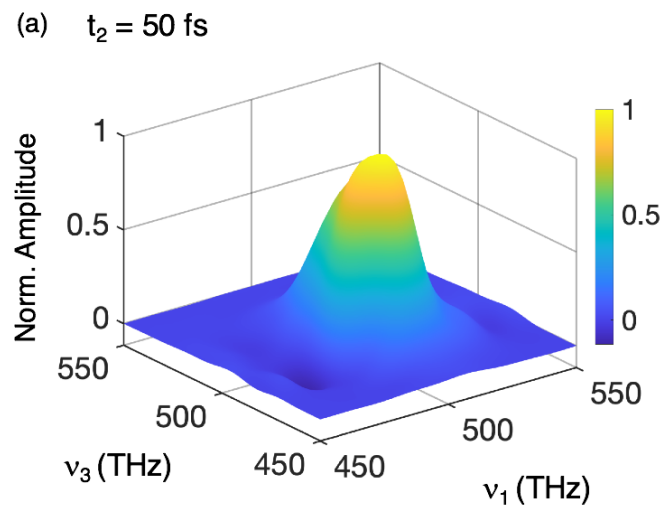

(c)
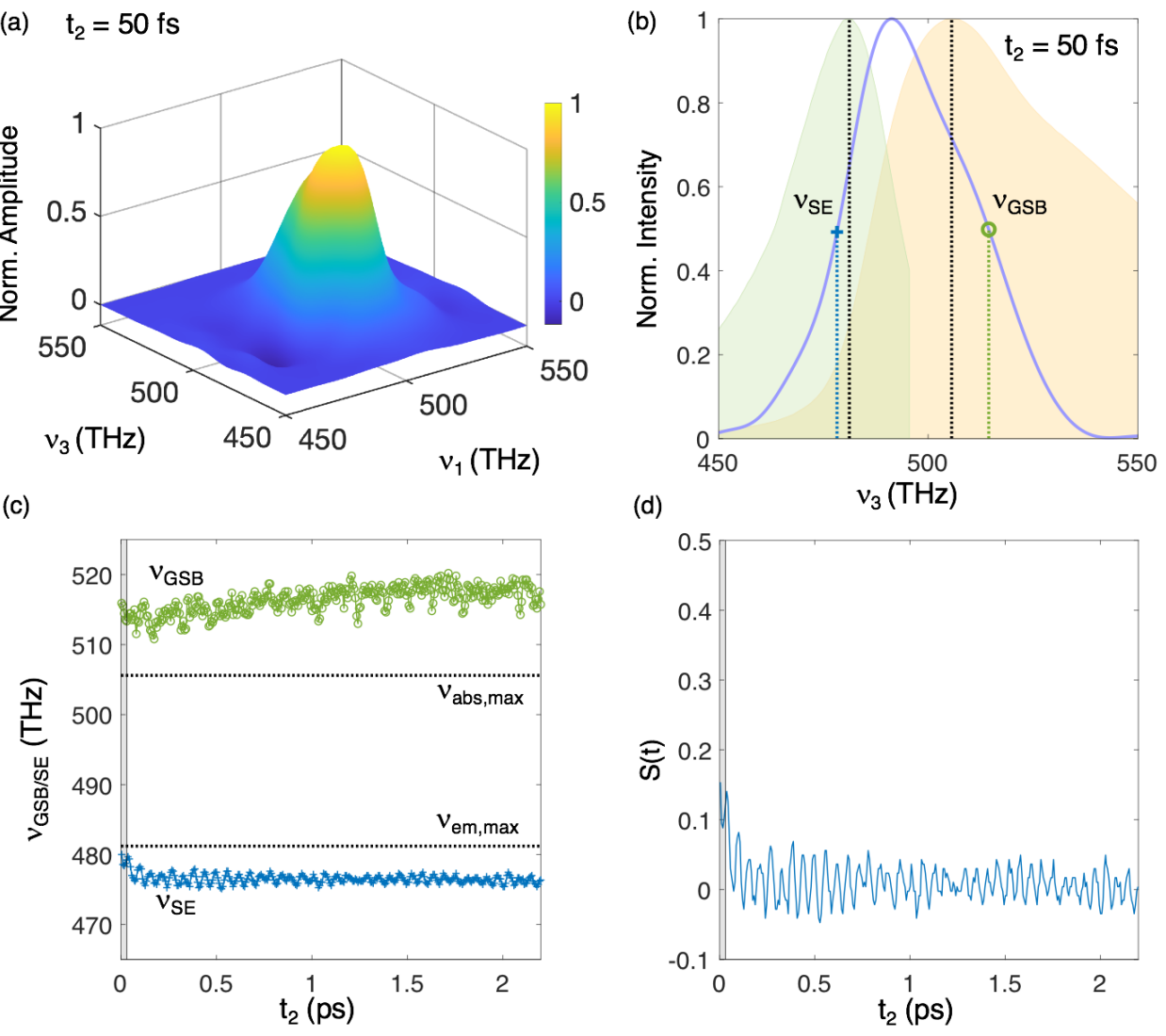

Figure S7. (a) Representative 2DES spectrum of cresyl violet at $t_{2}=50 \mathrm{fs}$. (b) Projection of the $t_{2}=50 \mathrm{fs} 2 \mathrm{DES}$ spectrum onto the $v_{3}$ axis (blue line) and the linear absorption (orange shaded area) and emission (light green shaded area) spectra. The spectra and projection were normalized for comparison. (c) $v_{\mathrm{SE}}$ (blue) and $v_{\mathrm{GSB}}$ (green) are plotted as a function of $\mathrm{t}_{2}$ time from 0 fs to $2.2 \mathrm{ps}$. The grey area (from $0 \mathrm{fs}$ up to $28 \mathrm{fs}$ ) indicates the time period when contributions from the coherent artifact may be present due to the pulse overlap. Data points within this time range are not included in the fitting process. (d) The Stokes shift function (S(t)) extracted from the projection of the 2DES spectra onto the $v_{3}$ axis. 
The dynamic Stokes shift $(\mathrm{S}(\mathrm{t}))$ is obtained from Eq. 1 of the main text taking $v(t)=v_{\operatorname{SE}}\left(t_{2}\right)$ for $t_{2}$ times ranging from 0 fs to $2.2 \mathrm{ps} . v(\infty)$ is taken as the average value of $v_{\mathrm{SE}}\left(\mathrm{t}_{2}\right)$ from 2 ps to $2.2 \mathrm{ps}$, (the last $200 \mathrm{fs}$ ). The denominator $(v(0)-v(\infty))$ is equivalent to the steady state Stokes shift obtained from the difference between the maxima of the linear absorption and fluorescence spectra, $24 \mathrm{THz}$ for cresyl violet in methanol. The extracted $\mathrm{S}(\mathrm{t})$ is plotted in Figure $\mathrm{S} 7 \mathrm{~d}$ as a function of $\mathrm{t}_{2}$ ranging from 0 fs to $2.2 \mathrm{ps}$.

To determine the timescale for the inertial solvent response, the Stokes shift function $\mathrm{S}(\mathrm{t})$ is modeled with a single Gaussian and single exponential function to account for the inertial and diffusive response of methanol. ${ }^{5-8}$ We note that we do not analyze the first 28 fs to exclude decays arising from the coherent artifacts. The functional forms for the gaussian and exponential fitting functions are shown below in Eq. S1. The fitting was performed by code written in MATLAB using the nonlinear least squares fitting algorithm (1sqnonlin function). The optimized values for the varied parameters are reported in Table 2 of the main manuscript and Table S4 in SI. Table S4 also reports the initial guesses and upper and lower boundary limits for the parameters, $A_{\text {gau }}, A_{\text {exp }}$, $\tau_{\text {gau }}$ and $\tau_{\text {exp. }} \tau_{\text {exp }}$ is set as a constant due to the limited $t_{2}$ time probed. A constant of $\tau_{\text {exp }}=1 \mathrm{ps}$ was used to be consistent with previous studies performed on similar chromophores in methanol. ${ }^{7}$ The error bars indicate $95 \%$ confidence intervals.

$$
\begin{array}{lc}
S(t) \stackrel{\text { fitting }}{\Longleftarrow} f_{1}(t)+f_{2}(t) & \text { Eq. } \mathrm{S} 1 \\
\text { where, } f_{1}(t)=A_{\text {gau }} e^{-\frac{t^{2}}{2 \tau_{\text {gau }}^{2}}} \text { and } f_{2}(t)=A_{\text {exp }} e^{-\frac{t}{\tau_{\text {exp }}}} \quad \text { Eq. S1 a-b }
\end{array}
$$

Table S4. The initial guesses with lower and upper boundary limits and the optimized parameters for fitting S(t) with a Gaussian and single exponential function (Eq. S1). Error bars report 95\% confidence intervals.

\begin{tabular}{|c|c|c|c|c|}
\hline & \multicolumn{2}{|c|}{ Initial Parameter Guesses and Boundary Limits } & \multirow{2}{*}{ Fitting Result } \\
\hline & Initial Guess & Lower Boundary & Upper Boundary & \\
\hline $\boldsymbol{A}_{\text {gau }}$ & 0.5 & 0 & 1 & $0.219 \pm 0.045$ \\
\cline { 1 - 4 } $\boldsymbol{\tau}_{\text {gau }}(\mathbf{f s})$ & 100 & 0 & 1000 & $29 \pm 3$ \\
\hline $\boldsymbol{A}_{\text {exp }}$ & 0.05 & 0 & 1 & $0.020 \pm 0.005$ \\
\hline $\boldsymbol{\tau}_{\text {exp }}(\mathbf{p s})$ & - & - & - & \multirow{2}{*}{1 (set as a constant) } \\
\hline
\end{tabular}


The resulting Gaussian and exponential fit is plotted in Figure S8a as a magenta line along with the experimentally obtained $S(t)$ (blue). Subtracting the fit from $S(t)$ yields the residuals, $R_{S(t)}$. The residuals are plotted in Figure S8b and oscillations arise from vibrational coherences. A Fourier transform of $R_{S(t)}$ yields four oscillatory components, $342 \mathrm{~cm}^{-1}, 523 \mathrm{~cm}^{-1}, 566 \mathrm{~cm}^{-1}$ and $589 \mathrm{~cm}^{-1}$, as shown in Fig S8c. Comparison with the calculated dipole strengths associated with the FC transitions (orange vertical lines) confirms the assignment to vibrational coherences. The details of assignment of FC active modes are described in Section 2.4 and Figure S6 of Supporting Information.
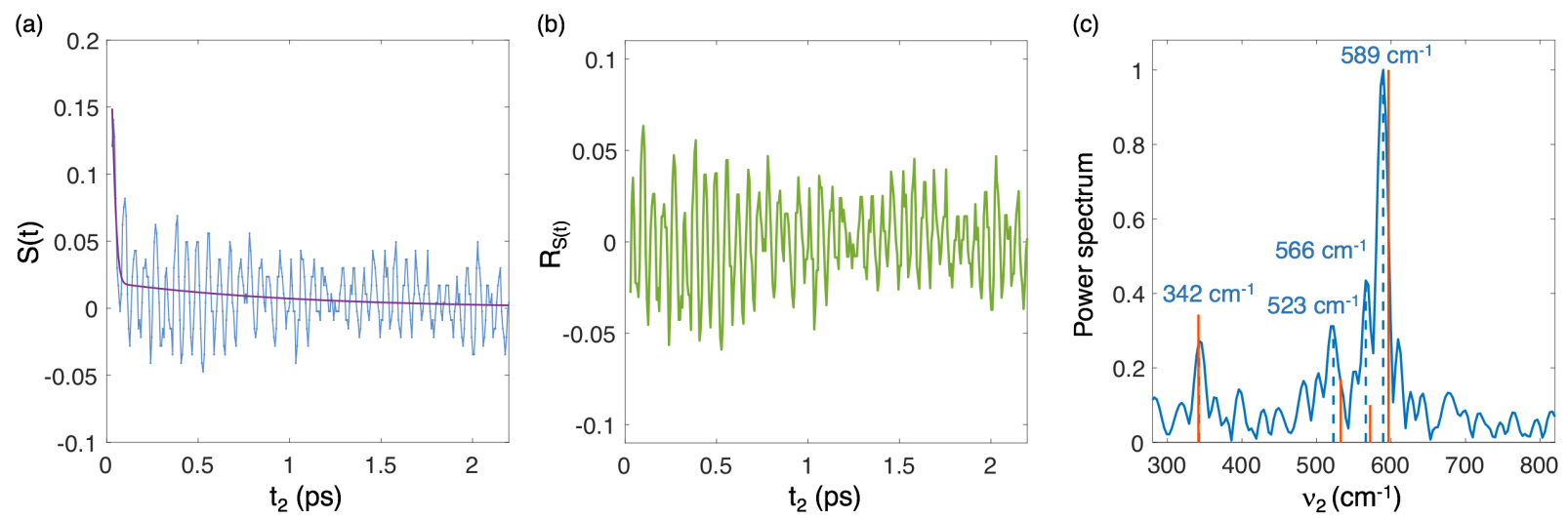

Figure S8. (a) The Stokes shift function $\mathrm{S}(\mathrm{t})$ (blue) along with the fit from a Gaussian and single exponential

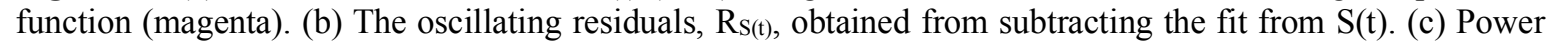
spectrum (blue) from Fourier transforming the residuals. Four peaks are observed and marked with blue dashed vertical lines and overlaid with the calculated Franck-Condon factors for absorption (orange vertical lines).

To account for the amplitude modulations resulting from vibrational coherences we have included four oscillatory components (based on the analysis of the residuals) in the fitting function along with the solvent response function. When the oscillatory components are included, the fitting function used to describe $\mathrm{S}(\mathrm{t})$ is given by Eq. $\mathrm{S} 2$, where $f_{1}(\mathrm{t})$ is given by Eq. S1a, $f_{2}(\mathrm{t})$ by Eq. S1b and $f_{v i b}(\mathrm{t})$ is given by Eq. S2a.

$$
\begin{aligned}
& S(t) \stackrel{f i t t i n g}{\Longleftarrow} f_{1}(t)+f_{2}(t)+\sum_{i}^{4} f_{v i b, i}(t) \\
& f_{v i b, i}(t)=A_{v i b, i} \cos \left(2 \pi v_{i} t-\varphi_{i}\right) e^{-\frac{t}{\tau_{v i b, i}}}
\end{aligned}
$$

The initial guess for the frequency of the four oscillatory modes comes from the Fourier transform of the residuals (Figure S8c), $342 \mathrm{~cm}^{-1}, 523 \mathrm{~cm}^{-1}, 566 \mathrm{~cm}^{-1}$ and $589 \mathrm{~cm}^{-1}$. The upper and lower boundaries for the frequencies are set to be within $\pm 10 \mathrm{~cm}^{-1}$ of the initial guesses. The dephasing times are estimated from the bandwidth of the peaks in the power spectrum. The FWHM of the peaks corresponds to a dephasing time of $1 \sim 2 \mathrm{ps}$. The lower boundaries for the dephasing time were set to $0.5 \mathrm{ps}$. 
We note that the fitting procedure is sensitive to the initial guess for the phase of the vibrational coherences. The initial phase parameters were chosen so that they qualitatively agree with the phase of the first few periods of the oscillations in the $\mathrm{S}(\mathrm{t})$ function. This is required in order to prohibit the Gaussian component from only describing the first half period of the initial oscillation. In Figure S9 we demonstrate how the fit results change with different initial guess conditions for the phase. The fitting results obtained from two different initial phase guesses are shown Figure S9b and Figure S9c. When the phase is chosen incorrectly, the Gaussian component only describes the first half period of the initial oscillation. The initial phase guess is chosen such that the Gaussian component bisects the oscillations as is shown in Figure S9b.
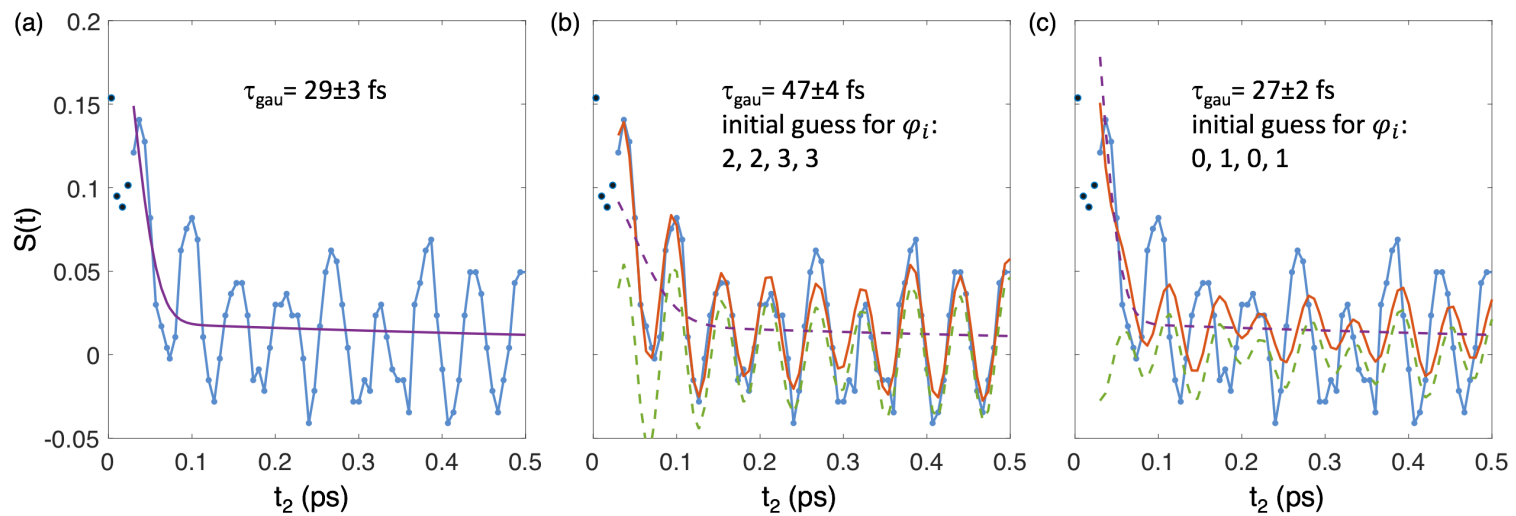

Figure S9. (a) The Stokes shift function $S(t)$ (blue) along with the fit from a single Gaussian and single exponential function (magenta). (b,c) The Stokes shift function $\mathrm{S}(\mathrm{t})$ (blue) along with the total fit (red) from a Gaussian and single exponential function (dashed, magenta) along with four damped cosines functions (dashed, green). (b) and (c) differ in the initial guess for the phases of the oscillations, where the guesses are denoted in the figures. 
The results of the best fit are reported in Table S5 along with the initial guesses and their upper and lower boundaries. The same fitting procedure was also applied to the slices taken at different $v_{1}$ frequencies to extract the frequency dependent ultrafast solvent response times. The extracted parameters taken at four representative slices, $v_{1}=490,500,510$ and $520 \mathrm{THz}$, are reported in Table S6. The initial guess for the phase of the vibrational coherences is listed in the parenthesis after reporting the fit result. From analysis for the $\mathrm{R}_{\mathrm{s}(\mathrm{t})}$ residuals, we find that the $342 \mathrm{~cm}^{-1}$ mode is less prominent and the $676 \mathrm{~cm}^{-1}$ is more prominent for slices taken at higher $v_{1}$ frequencies. This is consistent with the displaced oscillator model. ${ }^{9-13}$

Table S5. The initial guesses and optimized parameters for a Gaussian, single exponential, and four damped cosines for the fitting of $S(t)$ extracted from the projection of the 2DES spectra onto the $v_{3}$ axis.

\begin{tabular}{|c|c|c|c|c|}
\hline & Initial Value & Lower Boundary & Upper Boundary & Fitting Result \\
\hline$A_{g a u}$ & 0.5 & 0 & 1 & $0.094 \pm 0.011$ \\
\hline$\tau_{g a u}(\mathbf{f s})$ & 100 & 0 & 1000 & $47 \pm 4$ \\
\hline$A_{\text {exp }}$ & 0.05 & 0 & 1 & $0.018 \pm 0.002$ \\
\hline $\boldsymbol{\tau}_{\exp }(\mathbf{p s})$ & - & - & - & 1 (set as a constant) \\
\hline$A_{v i b, i}{ }^{a}$ & 0.01 & 0 & 1 & $\begin{array}{l}0.006 \pm 0.002 \\
0.012 \pm 0.002 \\
0.030 \pm 0.004 \\
0.027 \pm 0.002\end{array}$ \\
\hline$\tau_{v i b, i}(\mathbf{p s})^{a}$ & 2 & 0.5 & 10 & $\begin{array}{c}10.0 \pm 20.5 \\
2.9 \pm 1.3 \\
0.6 \pm 0.1 \\
7.7 \pm 3.0\end{array}$ \\
\hline$v_{i}\left(\mathrm{~cm}^{-1}\right)^{a}$ & $\begin{array}{l}342 \\
523 \\
566 \\
589\end{array}$ & $\begin{array}{l}332 \\
513 \\
556 \\
579\end{array}$ & $\begin{array}{l}352 \\
533 \\
576 \\
599\end{array}$ & $\begin{array}{l}344 \pm 1 \\
521 \pm 1 \\
567 \pm 2 \\
585 \pm 0\end{array}$ \\
\hline$\varphi_{i}^{a}$ & $\begin{array}{l}2 \\
2 \\
3 \\
3\end{array}$ & 0 & $2 \pi$ & $\begin{array}{c}0 \pm 0.25 \\
4.58 \pm 0.16 \\
3.44 \pm 0.14 \\
4.66 \pm 0.06\end{array}$ \\
\hline
\end{tabular}

a) The reported values are arranged in increasing $i$ index. 
Table S6. The fitting results of a Gaussian and single exponential combined with four damped cosine functions for fitting of the $\mathrm{S}(\mathrm{t})$ function extracted from analysis of slices taken at different $v_{1}$ frequencies.

\begin{tabular}{|c|c|c|c|c|}
\hline & $v_{1}=490 \mathrm{THz}$ & $v_{1}=500 \mathrm{THz}$ & $v_{1}=510 \mathrm{THz}$ & $v_{1}=520 \mathrm{THz}$ \\
\hline$A_{g a u}$ & $0.034 \pm 0.009$ & $0.104 \pm 0.010$ & $0.160 \pm 0.038$ & $0.090 \pm 0.019$ \\
\hline$\tau_{g a u}(\mathbf{f s})$ & $98 \pm 26$ & $52 \pm 4$ & $40 \pm 7$ & $60 \pm 10$ \\
\hline$A_{\text {exp }}$ & $0.023 \pm 0.003$ & $0.023 \pm 0.002$ & $0.005 \pm 0.006$ & $0.006 \pm 0.005$ \\
\hline$\tau_{\text {exp }}(\mathbf{p s})$ & 1 (set as a constant) & 1 (set as a constant) & 1 (set as a constant) & 1 (set as a constant) \\
\hline \multirow{4}{*}{$A_{v i b, i}^{a}$} & $0.027 \pm 0.006$ & $0.020 \pm 0.002$ & $0.022 \pm 0.006$ & $0.026 \pm 0.004$ \\
\hline & $0.020 \pm 0.002$ & $0.025 \pm 0.002$ & $0.040 \pm 0.006$ & $0.058 \pm 0.009$ \\
\hline & $0.010 \pm 0.004$ & $0.084 \pm 0.004$ & $0.066 \pm 0.011$ & $0.158 \pm 0.006$ \\
\hline & $0.107 \pm 0.004$ & $0.042 \pm 0.002$ & $0.167 \pm 0.007$ & $0.040 \pm 0.007$ \\
\hline \multirow{4}{*}{$\tau_{v i b, i}(\mathbf{p s})^{a}$} & $0.6 \pm 0.2$ & $1.4 \pm 0.3$ & $2.0 \pm 1.3$ & $3.0 \pm 1.4$ \\
\hline & $10.0 \pm 10.6$ & $2.4 \pm 0.5$ & $3.0 \pm 1.2$ & $0.5 \pm 0.1$ \\
\hline & $1.3 \pm 0.9$ & $0.8 \pm 0.0$ & $0.7 \pm 0.2$ & $1.0 \pm 0.1$ \\
\hline & $1.6 \pm 0.1$ & $10.0 \pm 3.3$ & $1.6 \pm 0.1$ & $1.0 \pm 0.2$ \\
\hline \multirow{4}{*}{$v_{i}\left(\mathrm{~cm}^{-1}\right)^{a}$} & $342 \pm 3$ & $338 \pm 1$ & $346 \pm 2$ & $520 \pm 1$ \\
\hline & $522 \pm 1$ & $520 \pm 0$ & $521 \pm 1$ & $565 \pm 2$ \\
\hline & $573 \pm 3$ & $572 \pm 0$ & $565 \pm 2$ & $586 \pm 0$ \\
\hline & $586 \pm 0$ & $579 \pm 0$ & $586 \pm 0$ & $666 \pm 1$ \\
\hline \multirow{4}{*}{$\varphi_{i}^{a}$} & $2.23 \pm 0.24(2)$ & $4.48 \pm 0.14(2)$ & $0 \pm 0.29(2)$ & $0 \pm 0.16(2)$ \\
\hline & $0.74 \pm 0.13(2)$ & $3.62 \pm 0.09(2)$ & $4.14 \pm 0.14(2)$ & $4.67 \pm 0.16(2)$ \\
\hline & $0 \pm 0.41$ & $3.22 \pm 0.04(2)$ & $2.70 \pm 0.16(2)$ & $0 \pm 0.04(2)$ \\
\hline & $0 \pm 0.04$ & $1.93 \pm 0.04(2)$ & $3.89 \pm 0.04(2)$ & $5.30 \pm 0.17(6)$ \\
\hline
\end{tabular}

a) The reported values are arranged in increasing $i$ index. 


\section{2DES of Cresyl Violet Obtained Under Different Pump Pulse Tunings}

To test the robustness of the analysis procedure we collected 2DES spectra of cresyl violet in methanol obtained under different spectral tunings of the incoming pump and probe pulses. The description of the pulse tunings, data sets, and extracted timescales for ultrafast solvation are reported below.

\subsection{Pulse Characterization}

For the additional 2DES data set the pump pulse has been tuned to the red, resulting in less spectral overlap with the linear absorption spectrum. Figure S10b displays the spectra of the pump (cyan line) and probe (brown line) pulses along with the absorption (orange shaded area) and fluorescence (light green shaded area) spectra. As shown in Figure S10a, the projection of the SFG-FROG onto the temporal axis for the additional data set has a FWHM of $19 \mathrm{fs}$.

(a)

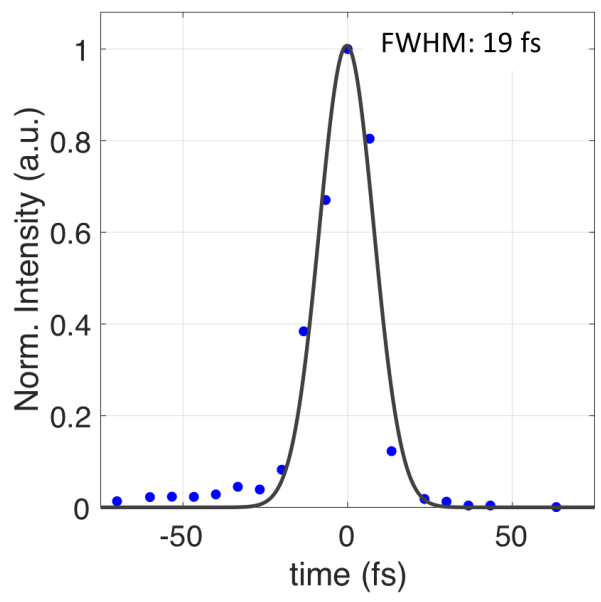

(b)

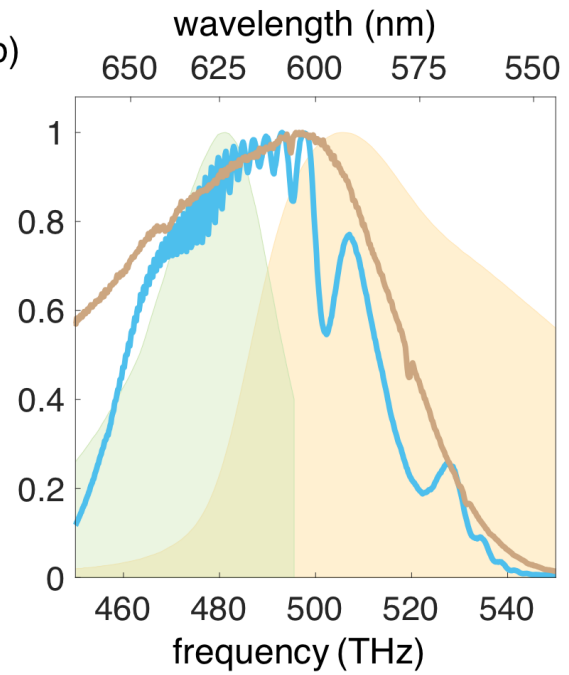

Figure S10. (a) Intensity of the SFG-FROG projected onto the time axis. (b) The spectra of the pump pulse after passing through the pulse shaper (cyan) and probe pulse (brown) used in the 2DES measurements. The linear absorption (orange) and emission spectra (light green) of cresyl violet dissolved in methanol are displayed as shaded areas. 


\subsection{DES Spectra and Amplitude Modulations}

Two representative 2DES spectra obtained under the different pulse tunings are shown in Figure S11a, b. The amplitude modulations at three coordinates are reported in Figure S11c.
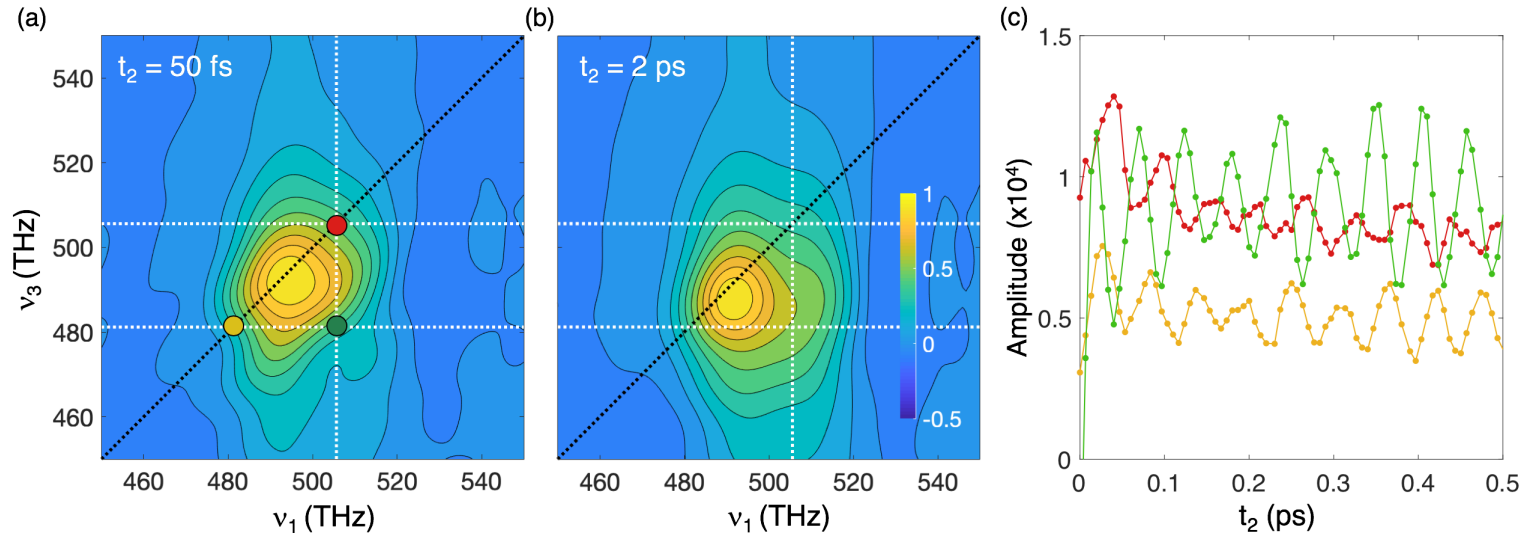

Figure S11. $(a, b)$ Representative absorptive 2DES spectra of cresyl violet dissolved in methanol for two different waiting times: $\mathrm{t}_{2}=50 \mathrm{fs}$ and $2 \mathrm{ps}$. The dashed line along the $v_{1}$ axis indicates the maximum of the absorption spectrum $(505 \mathrm{THz})$ and the two dashed lines along the $v_{3}$ axis indicate the maximum in the absorption (505 THz) and emission (481 THz) spectra, respectively. (c) The waiting time dependent amplitudes at three different $\left(v_{1}, v_{3}\right)$ coordinates: $v_{1}, v_{3}=505,505 \mathrm{THz}(\mathrm{red}) ; v_{1}, v_{3}=481,481 \mathrm{THz}$ (yellow), and $v_{1}, v_{3}=505,481 \mathrm{THz}$ (green), are shown. The coordinates are also denoted as colored dots on the $t_{2}=50 \mathrm{fs}$ 2DES spectrum. 


\subsection{Experimental Vibrational Coherences}

The experimentally extracted power spectra from the 2DES spectra obtained under different pulse tuning conditions are presented in Figure S12 along with the calculated vibrational modes (see calculation details in section 2 of the Supporting Information). For this pulse tuning, we extract seven vibrational coherences, indicated as dashed blue vertical lines at 340,469, 489, 520, 570, 590 and $677 \mathrm{~cm}^{-1}$ from the averaged power spectrum. Compared to the modes extracted under the pulse tuning in the main text, one extra mode at $489 \mathrm{~cm}^{-1}$ is reported here. This mode is attributed to a ground state vibrational coherence according to the non-resonant Raman calculation. Compared with the pulse tuning in the main text, tuning the pump pulse to have less overlap with the absorption spectrum leads to more vibrational coherences associated with the non-resonant Raman modes.

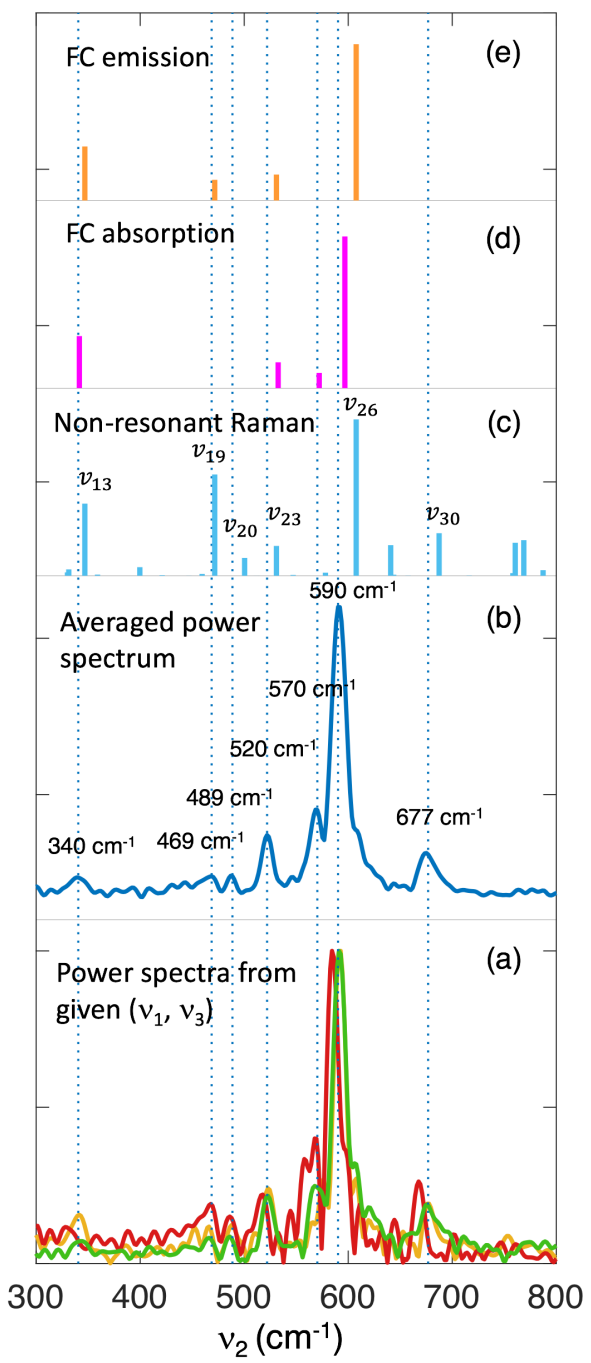

Figure S12. (a) The power spectra from Fourier transforming the residuals taken at three different $v_{1}, v_{3}$ coordinates: $v_{1}, v_{3}=505,505 \mathrm{THz}$ (red); $v_{1}, v_{3}=481,481 \mathrm{THz}$ (yellow), and $v_{1}, v_{3}=505,481 \mathrm{THz}$ (green). The coordinates are also indicated by colored dots on the 2D spectrum in Figure S11(a). (b) The average power spectrum for all $\left(v_{1}, v_{3}\right)$ coordinates in the 2DES spectra. (c) Calculated non-resonant Raman active modes of cresyl violet in methanol (cyan). (d) Franck-Condon active modes associated with the absorption (magenta). (e) Franck-Condon active modes associated with the emission (orange). 


\subsection{Extraction and Analysis of the Dynamic Stokes Shift from the 2DES Spectra}

The Stokes shift function is extracted from the 2DES spectra (from both the projection (Figure S13) and from slices taken at different $v_{1}$ frequencies (Figure S14)) according to the same procedure outlined in the main text and section 3 of the Supporting Information. The normalized projection is plotted in Figure S13a and $v_{\mathrm{SE}}$ and $v_{\mathrm{GSB}}$ were taken at $50 \%$ of the maximum amplitude. Over the course of the first $200 \mathrm{fs}$, a fast decay in $v_{\mathrm{SE}}$ is observed. Data points lying within the first 28 fs were excluded from the analysis due to both the behavior of $v_{\mathrm{GSB}}$ and the temporal duration
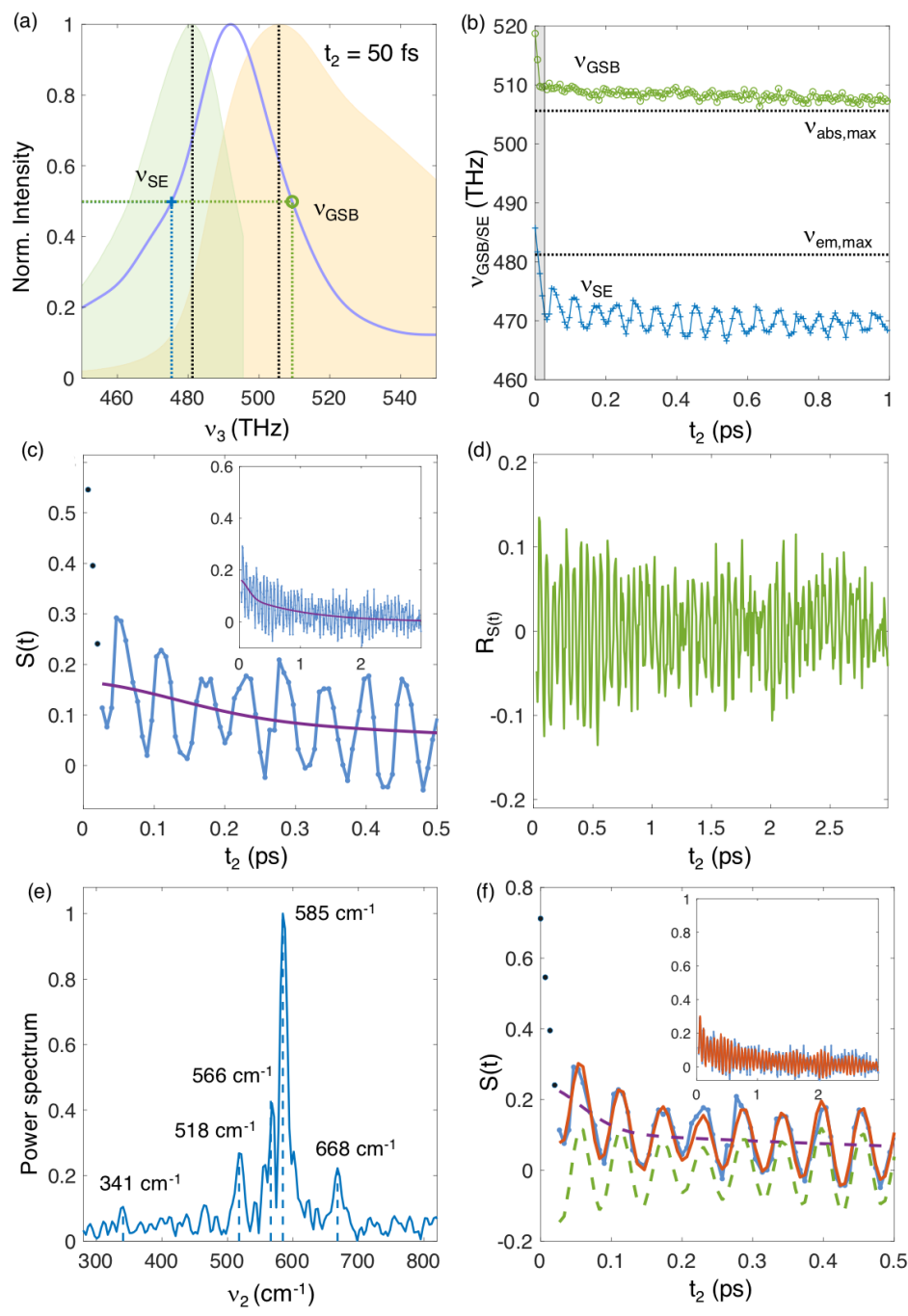

Figure S13. (a) Representative projection of the 2DES spectrum at $\mathrm{t}_{2}=50 \mathrm{fs}$ (blue line) and the linear absorption (orange shaded area) and emission (light green shaded area) spectra. (b) Plots of $v_{\mathrm{SE}}$ (blue) and $v_{\mathrm{GSB}}$ (green) as a function of $t_{2}$ time for the first 1 ps. (c) The Stokes shift function $S(t)$ (blue dots and line) from the projection of the 2DES spectra onto the $v_{3}$ axis and the corresponding fit from the sum of a Gaussian and single exponential decay function (magenta line). The inset plots $S(t)$ from 0 to 3 ps and the main plot zooms in on the first 500 fs. (d) The Rs(t) residuals. (e) The power spectrum obtained from Fourier transforming the Rs(t) residuals. Five prominent oscillatory modes are observed and marked by dashed vertical lines. (f) The Stokes shift function S(t) (blue dots and line) and corresponding fit from the sum of a Gaussian and single exponential decay along with five cosine functions is plotted as a red line. The magenta dashed line represents the sum of the Gaussian and single exponential decay function. The green dashed line indicates the sum of the four cosine functions. The inset plots $S(t)$ up to 3 ps and the main plot zooms in on the first $500 \mathrm{fs}$. 
of the laser pulse, 19 fs. The same procedures described in the main text and section 3 of the SI were applied to fit the Stokes shift function, $\mathrm{S}(\mathrm{t})$. With the different pulse tuning five oscillatory modes having frequencies of $341 \mathrm{~cm}^{-1}, 518 \mathrm{~cm}^{-1}, 566 \mathrm{~cm}^{-1}, 585 \mathrm{~cm}^{-1}$ and $668 \mathrm{~cm}^{-1}$ are observed in the residual, $\mathrm{R}_{\mathrm{S}(\mathrm{t})}$ (Figure $\mathrm{S} 13 \mathrm{e}$ ). These oscillatory components are included in the fitting procedure.
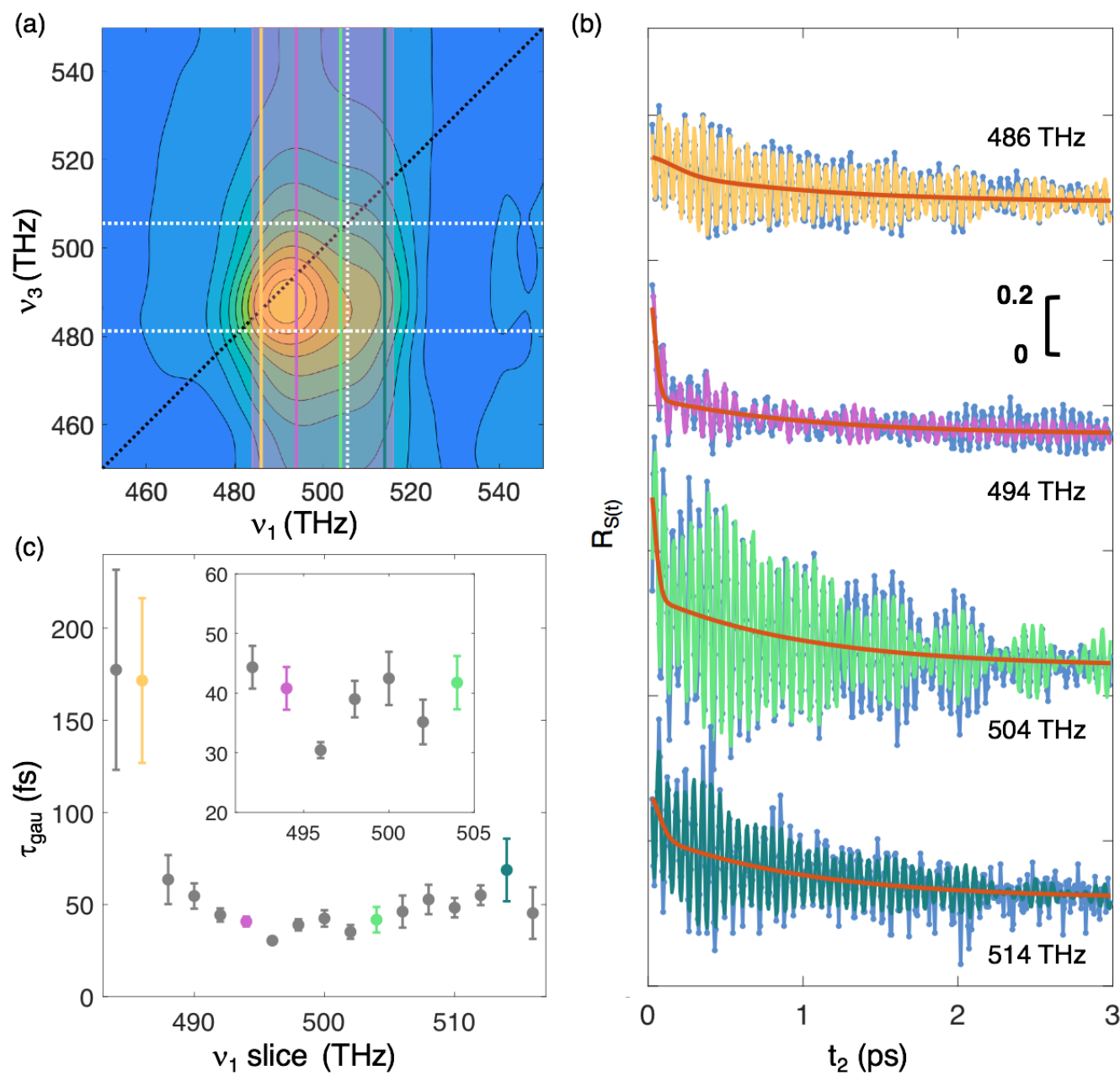

Figure S14. (a) The 2DES spectrum at $t_{2}=2$ ps is shown with a light red shaded box indicating the region over which $v_{1}$ slices were taken. The $v_{1}$ frequencies where four representative slices were taken are indicated as solid vertical lines at $v_{1}=486$ (yellow), 494 (magenta), 504 (light green) and 514 (dark green) THz. (b) The Stokes shift function $\mathrm{S}(\mathrm{t})$ (blue) along with corresponding fit for four representative slices is shown. The Stokes shift functions are shifted for better visual representation and the amplitude scale is indicated as a bar on the plot. The red lines indicate the Gaussian and single exponential contributions to the fit. (c) A plot of the extracted gaussian component as a function of the $v_{1}$ slice frequency is plotted. The inset plots a zoomed in region where the fastest timescales are observed.

The optimized parameters obtained from analyzing the projection and slices are reported in Table S7. The trend of $\tau_{\text {gau }}$ under different pulse tuning is similar to that in the data set presented in the main text. The Gaussian time component near the center of the peak in the 2DES spectrum has the fastest timescale compared to Gaussian components extracted from the lower and higher $v_{1}$ frequency regions. In comparing the extracted timescales of the Gaussian component, we find similar results with a fastest timescale of $\sim 35$ fs attributed to the inertial component. 
Table S7. The optimized fit parameters for $\mathrm{S}(\mathrm{t})$ extracted from 2DES spectra obtained under different pulse tunings.

\begin{tabular}{|c|c|c|c|c|c|c|}
\hline & Projection & Projection & $v_{1}=486 \mathrm{THz}$ & $v_{1}=494 \mathrm{THz}$ & $v_{1}=504 \mathrm{THz}$ & $v_{1}=514 \mathrm{THz}$ \\
\hline$A_{g a u}$ & $0.059 \pm 0.013$ & $0.129 \pm 0.022$ & $0.046 \pm 0.011$ & $0.388 \pm 0.033$ & $0.422 \pm 0.090$ & $0.140 \pm 0.038$ \\
\hline$\tau_{g a u}(\mathbf{f s})$ & $135 \pm 31$ & $56 \pm 8$ & $172 \pm 45$ & $41 \pm 3$ & $42 \pm 7$ & $69 \pm 17$ \\
\hline$A_{\text {exp }}$ & $0.106 \pm 0.006$ & $0.112 \pm 0.005$ & $0.113 \pm 0.006$ & $0.128 \pm 0.006$ & $0.246 \pm 0.016$ & $0.224 \pm 0.011$ \\
\hline$\tau_{\text {exp }}(\mathbf{p s})$ & 1 (constant) & 1 (constant) & 1 (constant) & 1 (constant) & 1 (constant) & 1 (constant) \\
\hline \multirow{5}{*}{$A_{v i b, i}{ }^{a}$} & & $0.015 \pm 0.006$ & $0.026 \pm 0.007$ & $0.070 \pm 0.011$ & $0.058 \pm 0.019$ & $0.008 \pm 0.007$ \\
\hline & & $0.020 \pm 0.004$ & $0.028 \pm 0.005$ & $0.040 \pm 0.005$ & $0.062 \pm 0.011$ & $0.035 \pm 0.009$ \\
\hline & & $0.018 \pm 0.003$ & $0.018 \pm 0.005$ & $0.093 \pm 0.012$ & $0.373 \pm 0.034$ & $0.017 \pm 0.007$ \\
\hline & & $0.096 \pm 0.005$ & $0.177 \pm 0.006$ & $0.039 \pm 0.004$ & $0.390 \pm 0.020$ & $0.261 \pm 0.014$ \\
\hline & & $0.017 \pm 0.004$ & $0.014 \pm 0.004$ & $0.011 \pm 0.004$ & $0.040 \pm 0.013$ & $0.046 \pm 0.011$ \\
\hline \multirow{5}{*}{$\tau_{v i b, i}(\mathbf{p s})^{\mathrm{a}}$} & & $1.6 \pm 1.1$ & $1.3 \pm 0.5$ & $0.6 \pm 1.1$ & $1.6 \pm 0.8$ & $9.6 \pm 51.3$ \\
\hline & & $6.9 \pm 5.6$ & $3.6 \pm 1.6$ & $2.5 \pm 0.7$ & $10.0 \pm 10.9$ & $2.9 \pm 1.8$ \\
\hline & & $10.0 \pm 11.6$ & $3.5 \pm 2.3$ & $0.5 \pm 0.1$ & $0.5 \pm 0.1$ & $10.0 \pm 26.8$ \\
\hline & & $2.7 \pm 0.3$ & $2.1 \pm 0.1$ & $10.0 \pm 6.1$ & $1.4 \pm 0.1$ & $1.2 \pm 0.1$ \\
\hline & & $4.4 \pm 3.3$ & $9.1 \pm 14.2$ & $10.0 \pm 21.8$ & $3.6 \pm 3.2$ & $2.0 \pm 0.8$ \\
\hline \multirow{5}{*}{$v_{i}\left(\mathrm{~cm}^{-1}\right)^{\mathrm{a}}$} & & $341 \pm 2$ & $345 \pm 2$ & $348 \pm 2$ & $342 \pm 2$ & $345 \pm 3$ \\
\hline & & $522 \pm 1$ & $526 \pm 1$ & $520 \pm 1$ & $521 \pm 1$ & $525 \pm 1$ \\
\hline & & $570 \pm 1$ & $564 \pm 1$ & $563 \pm 2$ & $576 \pm 1$ & $569 \pm 1$ \\
\hline & & $586 \pm 0$ & $590 \pm 0$ & $595 \pm 0$ & $590 \pm 0$ & $589 \pm 0$ \\
\hline & & $666 \pm 1$ & $666 \pm 1$ & $662 \pm 1$ & $668 \pm 1$ & $665 \pm 1$ \\
\hline \multirow{5}{*}{$\varphi_{i}^{\mathrm{a}}$} & & $0 \pm 0.39$ & $3.80 \pm 0.28(2)$ & $0 \pm 0.16(2)$ & $0 \pm 0.32(2)$ & $0 \pm 0.88$ \\
\hline & & $0.05 \pm 0.18$ & $3.00 \pm 0.17(2)$ & $4.78 \pm 0.14(2)$ & $5.30 \pm 0.17(2)$ & $2.19 \pm 0.27(2)$ \\
\hline & & $6.09 \pm 0.19(2)$ & $0.01 \pm 0.26(2)$ & $3.55 \pm 0.13(2)$ & $3.75 \pm 0.09(2)$ & $6.28 \pm 0.41(2)$ \\
\hline & & $0 \pm 0.05$ & $1.98 \pm 0.03(2)$ & $4.76 \pm 0.10$ & $0 \pm 0.05$ (2) & $1.40 \pm 0.05(2)$ \\
\hline & & $6.14 \pm 0.23(6)$ & $6.28 \pm 0.27(6)$ & $4.22 \pm 0.35(6)$ & $5.05 \pm 0.33(6)$ & $6.28 \pm 0.24(6)$ \\
\hline
\end{tabular}

a) The reported values are arranged in increasing $i$ index. 


\section{Excitation Frequency Dependence of Spectral Diffusion from Model Spectra}

In addition to the dynamic Stokes shift spectral diffusion also contributes to the spectral line shape changes. Here we consider how spectral diffusion impacts the extracted value of $v_{\mathrm{SE}}\left(\mathrm{t}_{2}\right)$ for different $v_{1}$ slices. We first simulate the $2 \mathrm{D}$ spectra for a single transition using the Kubo lineshape. ${ }^{14}$ The Kubo lineshape does not account for relaxation of the $S_{1}$ state, allowing for the examination of the effects of spectral diffusion alone. Simulated 2D spectra at $\mathrm{t}_{2}$ times of $200 \mathrm{fs}$ and 5 ps are displayed in Figure S15a,b. The following parameters are used for the simulation: $\Delta \omega=9.5 \mathrm{THz}, \tau_{\mathrm{c}}=1 \mathrm{ps}$. At $200 \mathrm{fs}$, the peak is inhomogeneously broadened and elongated along the diagonal. As the waiting time increases the peak becomes more round as the inhomogeneity
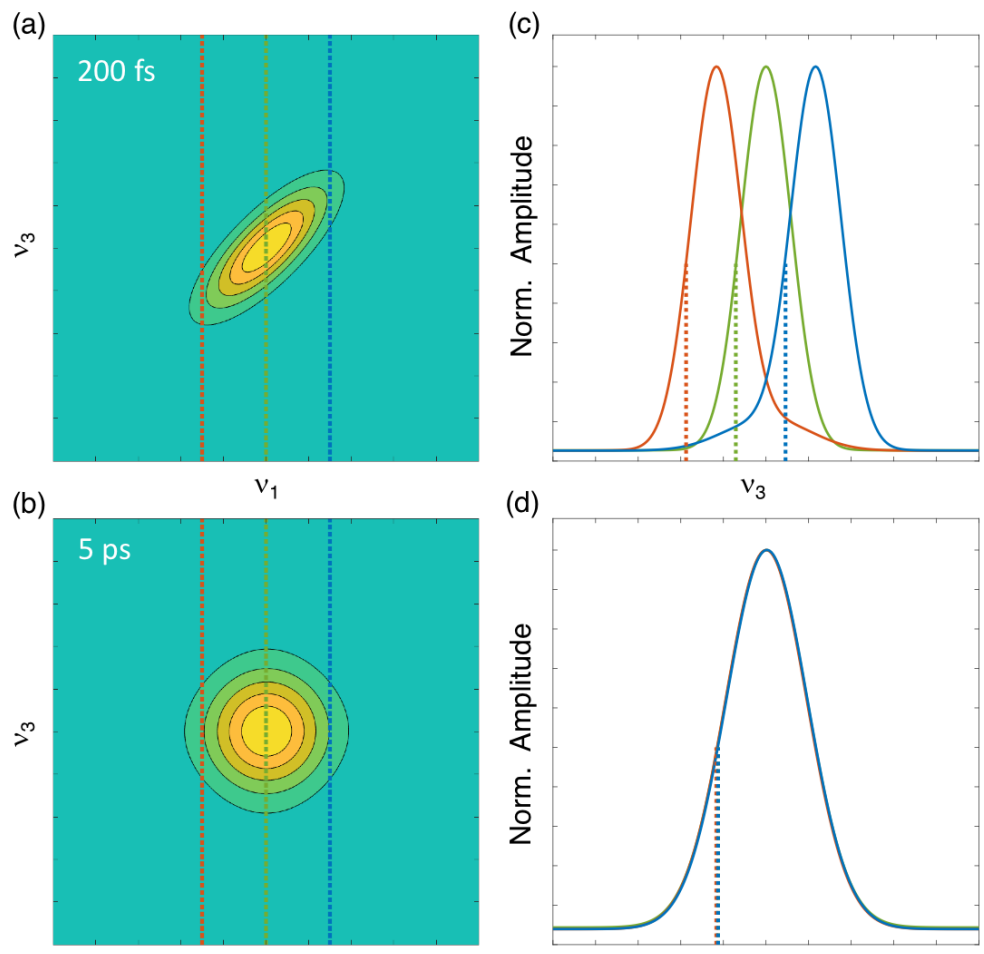

(e)

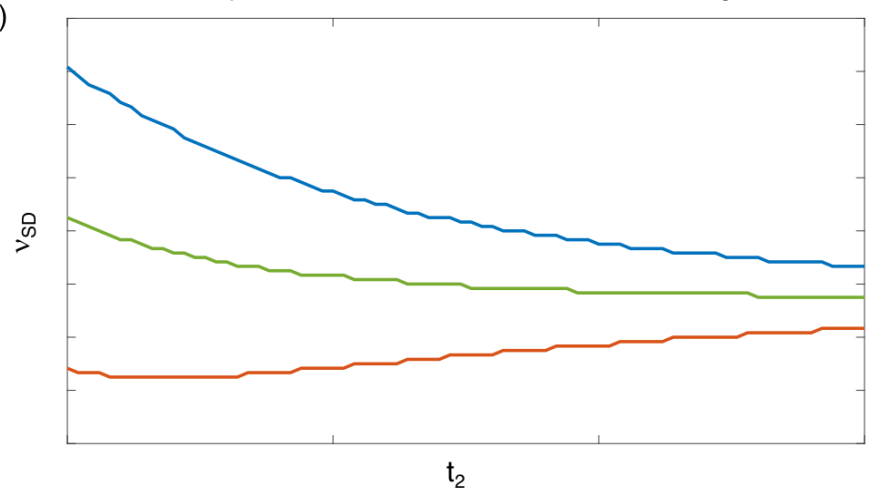

Figure S15. ( $a, b)$ The 2D spectrum simulated with the Kubo line shape model at $\mathrm{t}_{2}$ time $200 \mathrm{fs}$ and $5 \mathrm{ps}$. Three slices are taken at different $v_{1}$ frequencies marked with colored dashed lines. (c,d) The slices taken at different $v_{1}$ frequencies are plotted as a function of $v_{3}$ for the $2 \mathrm{D}$ spectra in $(\mathrm{a}, \mathrm{b})$, respectively. The position of $v_{\mathrm{SD}}$ is denoted by dashed lines. (e) The plot of $v_{\mathrm{SD}}$ from analysis of the three slices as a function of waiting time $\mathrm{t}_{2}$. 
decays. Normalized slices taken on the red edge (red line), blue edge (blue line), and center of the peak (green line) are plotted for in Figure S15c,d for 2D spectra at early and later waiting times. Note that the slices are normalized for comparison and this leads to an overlap in the slices at longer time delays.

Comparison of the representative slices in Figure S15 shows that the slices change as a function of $t_{2}$ time. To compare the $t_{2}$ dependence of the slices we extract $v_{S D}$, using the same procedure to extract $v_{\mathrm{SE}}$, where for a given $\mathrm{t}_{2}$ time we record the $v_{3}$ frequency at $50 \%$ of the maximum for the lower energy portion of the peak. For the Kubo lineshape, where only spectral diffusion is considered, we find that the extracted $v_{\mathrm{SD}}$ from the slices taken on the higher $v_{1}$ frequency side decay to lower frequencies, exhibiting a downward frequency shift as a function of $\mathrm{t}_{2}$ (blue line, Figure S15e). Slices taken from the center of the peak also display a downward shift to lower frequencies; however, the shift is less pronounced when compared to the slices taken on the blue edge. Analysis of slices taken from the lower $v_{1}$ frequency side (the red edge) results in $v_{\mathrm{SD}}$ shifts to higher frequencies (Figure S15e, red line). We note that the overall magnitude of the frequency shift, $\Delta v_{\mathrm{SD}}$ where $\Delta v_{\mathrm{SD}}=v_{\mathrm{SD}}(0)-v_{\mathrm{SD}}(5 \mathrm{ps})$, for slices taken at the center and on the red edge are similar - however the sign is opposite as $v_{\mathrm{SD}}$ extracted from the red edge leads to an eventual increase in frequency at longer $\mathrm{t}_{2}$ times.

The evolution of $v_{S D}$, which arises solely from spectral diffusion, will affect the extracted $v_{\mathrm{SE}} . v_{\mathrm{SE}}$ extracted from the lower energy $v_{1}$ slices will be offset by the increase in $v_{\text {SD. }} v_{\text {SE }}$ extracted from the higher energy $v_{1}$ slices will be enhanced by the larger decrease in $v_{\mathrm{SD}}$. $v_{\mathrm{SE}}$ extracted from $v_{1}$ slices taken at the center of the peak, will also be affected, but we expect the smaller $\Delta v_{\mathrm{SD}}$ value and the decaying feature will have less of an impact on $v_{\mathrm{SE}}\left(\mathrm{t}_{2}\right)$. To further investigate this we simulate the 2DES with a displaced oscillator model, ${ }^{15,16}$ where both spectral diffusion and $\mathrm{S}_{1}$ relaxation can occur. For the displaced oscillator model the parameters of the bath $\left(\omega=80 \mathrm{~cm}^{-1}, \gamma\right.$ $=200 \mathrm{~cm}^{-1}, \mathrm{~S}=5, \mathrm{~T}=298 \mathrm{~K}$ ) were set so that the Stokes shift is $24 \mathrm{THz}$. Representative simulated 2DES spectra are plotted in Figure S16 at waiting time $200 \mathrm{fs}$ and $3 \mathrm{ps}$. As $\mathrm{t}_{2}$ increases the system relaxes and the dynamic Stokes shift leads to an asymmetric line broadening along the $v_{3}$ axis. We extract $v_{\mathrm{SE}}$ from the simulated spectra from slices taken on the red edge (red), blue edge (blue), and at the center (green) of the peak. The extracted $\nu_{\mathrm{SE}}$ frequencies are plotted as a function of $\mathrm{t}_{2}$ in Figure S16.
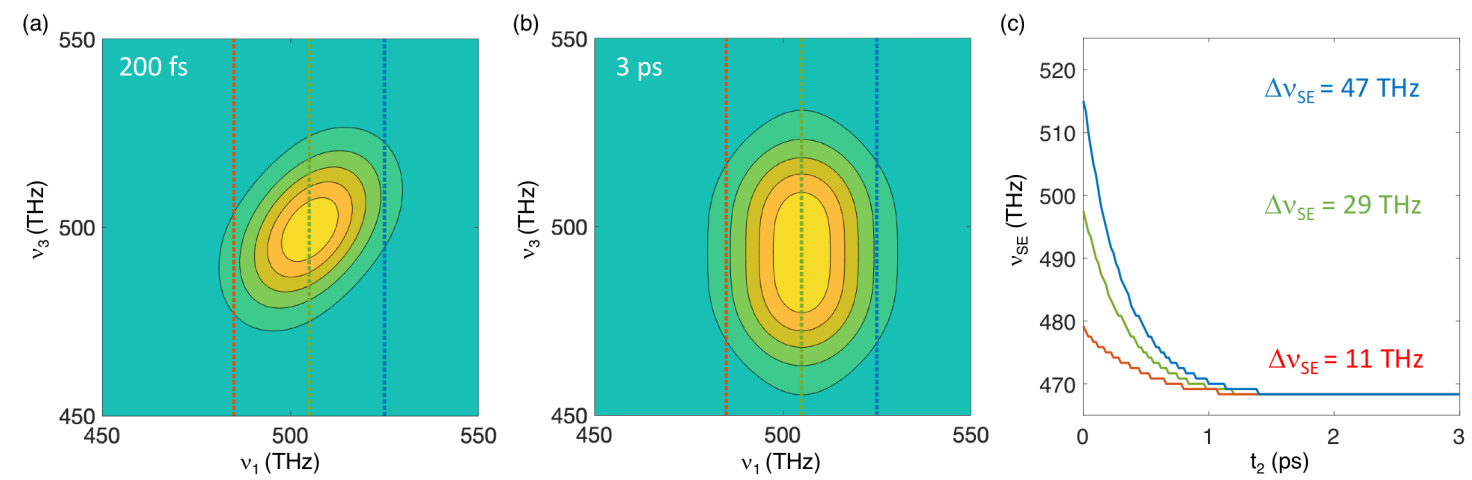

Figure S16. $(a, b)$ The 2D spectrum simulated with the displaced oscillator model at $t_{2}$ time $200 \mathrm{fs}$ and $3 \mathrm{ps}$. Slices are taken at three different $v_{1}$ frequencies marked with colored dashed lines. (c) The plot of $v_{\mathrm{SE}}$ extracted from the three different slices as a function of waiting time $t_{2}$. The magnitudes of the shift are denoted with the same color-scheme. 
For slices taken from the three different regions, $v_{\mathrm{SE}}$ taken near the center of the peak has the closest total frequency shift, $\Delta v_{\mathrm{SE}}=v_{\mathrm{SE}}(0)-v_{\mathrm{SE}}(3 \mathrm{ps})$ to the input Stokes shift. The red edge has a total frequency shift of $\Delta v_{\mathrm{SE}}=11 \mathrm{THz}$, much less than the input $24 \mathrm{THz}$. This is attributed to the offset arising from the positive frequency shift of $v_{\mathrm{SD}}$. The slices taken on the blue edge have an increased total shift of $\Delta v_{\mathrm{SE}}=47 \mathrm{THz}$ compared to the input $24 \mathrm{THz}$. This is attributed to the enhancement arising from the decay of $v_{\mathrm{SD}}$. Slices taken at the center have an overall frequency shift of $\Delta v_{\mathrm{SE}}=29 \mathrm{THz}$ which is closest to the $24 \mathrm{THz}$ input parameter. A comparison of the total frequency shifts $\Delta v_{\mathrm{SE}}$ further indicates that the line shape changes associated with spectral diffusion will be minimized by taking slices from the center of the peak. 


\section{Comparison of a Global Analysis and the Dynamic Stokes Shift}

In the main manuscript we have focused on extracting $v_{\mathrm{SE}}$, a frequency parameter associated with the stimulated emission, in order to obtain the dynamic Stokes shift. The motivation for extracting the dynamic Stokes shift is that this parameter can be mapped back to the solvation dynamics as it is equivalent to the normalized frequency fluctuation correlation function. ${ }^{5,6,17,18}$

In extracting $v_{\mathrm{SE}}$, we are focusing on analyzing the lower energy portion of the peaks along the $v_{3}$ axis in the 2DES spectra. The relaxation of the $S_{1}$ state leads to a decrease in the $S_{1}-S_{0}$ energy gap corresponding to a decrease in the transition frequency of the stimulated emission. In the 2DES spectra this relaxation appears as an asymmetric line broadening along the $v_{3}$ axis as the spectral feature associated with the stimulated emission shifts towards lower frequencies. A question arises as to whether a global analysis could also capture this line broadening/relaxation.

To investigate this we applied a global analysis to the slices taken at different $v_{1}$ frequencies. Here we modified our previous described procedure ${ }^{19,20}$ to include a Gaussian component in addition to exponential components allowing for a more direct comparison to the timescales extracted from the analysis of the dynamic Stokes shift. We note that we did not include oscillatory components in either analysis in this section, thus the extracted Gaussian timescales from analysis of the dynamic Stokes shift reported here differ from those in the main text. However, a comparison between the global analysis and dynamic Stokes shift can still be made as the data sets are analyzed in the same fashion.

For the global analysis we find that three components are required to fully describe the time dependence of the system and the decay associated spectra (DAS) and temporal components are plotted in Figure S17 for analysis of slices taken at three different $v_{1}$ excitation frequencies. To interpret the DAS, a negative amplitude in the DAS indicates a decay and a positive amplitude in
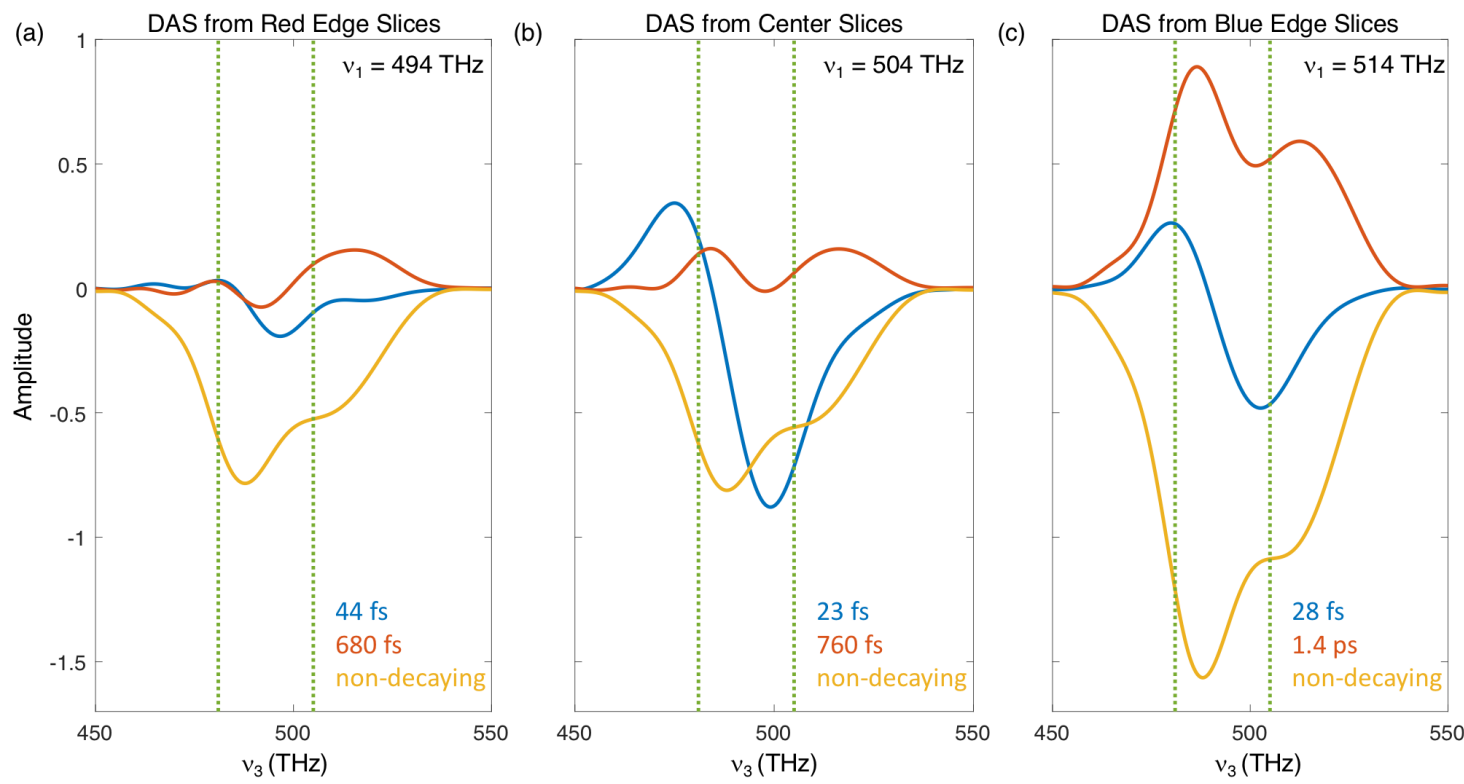

Figure S17. The decay associated spectra (DAS) extracted from slices taken at: (a) $v_{1}=494 \mathrm{THz}$ (b) $v_{1}=504$ $\mathrm{THz}$, and (c) $v_{1}=514 \mathrm{THz}$. The timescales of the temporal components are marked using the same color scheme. The positions of the ground state bleach $(505 \mathrm{THz})$ and stimulated emission $(481 \mathrm{THz})$ are denoted in dashed green lines. 
the DAS indicates a growth for spectral features associated with the ground state bleach and stimulated emission (negative signals). For all three slices, the longest timescale component is plotted in yellow and indicates an overall decay in the GSB and SE region on the few ps timescales. Note that we do not fully characterize this decay given the limited $t_{2}$ time scanned and it is denoted as non-decaying in the figure. The DAS plotted in red evolve on the $\sim 680$ fs to $1.4 \mathrm{ps}$ timescale depending on the $v_{1}$ frequency. The DAS plotted in blue are modeled with a Gaussian function and evolve on the 22-100 fs timescale depending on the $v_{1}$ frequency.

Focusing on the DAS obtained from analyzing slices taken at a $v_{1}$ frequency towards the center of the peak (Figure S17b) the DAS evolving on the $\sim 23 \mathrm{fs}$ timescale shows a growth in the stimulated emission region - which we attribute to the relaxation of the $\mathrm{S}_{1}$ state. In addition, we see decays in other spectral regions which may be attributed to frequency shifts as relaxation occurs or other lineshape changes that occur on this timescale. The DAS evolving on the 760 fs timescale has two main growth features on either side if the GSB feature. This is what we would expect for a system undergoing spectral diffusion, where a lineshape change would lead to an increase in the peak width above and below the diagonal. Comparing the global analysis for slices taken at different $v_{1}$ frequencies (Fig. 17a,c) - we find that the 22-100 fs growth in the stimulated emission region (blue trace) is more pronounced and has less contributions from other spectral features for slices taken at $v_{1}$ frequencies towards the center. This is consistent with our analysis of the $v_{1}$ frequency dependence discussed in section 5 of the Supporting Information and the main text.

We also compare the timescales of the Gaussian components extracted from the global analysis to the Gaussian components extracted from analysis of the dynamic Stokes shift (Eq. S1 in the main text) in Figure S18 for slices taken at different $v_{1}$ frequencies. Again, we note the Gaussian timescales reported in Figure S18 are not the same as those reported in the main manuscript as the oscillations are not accounted for here. The error bars report $95 \%$ confidence intervals. Comparing the Gaussian components extracted from the two analysis procedures yields similar trends, with increased timescales on the red and blue edge of the peaks and the fastest timescales extracted from slices taken at the center of the peak. Though a similar trend is observed, the timescales differ. The differences in timescales can be attributed to the fact that the global analysis extracts average
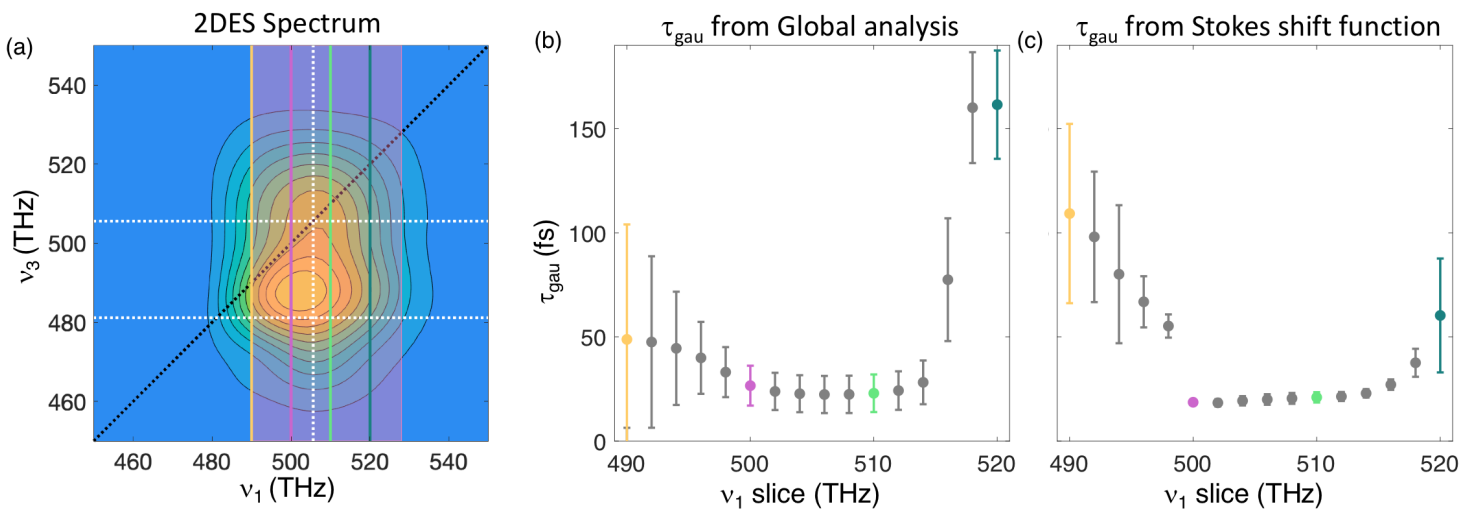

Figure S18. (a) The 2DES spectrum at $\mathrm{t}_{2}=2 \mathrm{ps}$ is shown with a light red shaded box indicating the region over which $v_{1}$ slices were taken. The representative $v_{1}$ frequencies are indicated as solid vertical lines at $v_{1}=490$ (yellow), 500 (magenta), 510 (light green) and 520 (dark green) THz. (b) The extracted gaussian component from the global analysis is plotted as a function of the $v_{1}$ slice frequency. (c) The extracted gaussian component from the Stokes shift function is plotted as a function of the $v_{1}$ slice frequency (note oscillatory components are not included here). For both (b) and (c) error bars represent 95\% confidence intervals. 
timescales associated with the growth and decay of spectral components, while the dynamic Stokes shift focuses on mapping the frequency shift associated with the relaxation of the $\mathrm{S}_{1}$ state through monitoring the lower energy portion of the peak associated with the stimulated emission. Future work will focus on a more thorough comparison of these two different techniques. For the current work we focus on analyzing the dynamic Stokes shift given its established relationship to the frequency fluctuation correlation function. ${ }^{5,6,17,18}$ 


\section{References}

(1) DeLong, K. W.; Trebino, R.; Hunter, J.; White, W. E. Frequency-Resolved Optical Gating with the Use of Second-Harmonic Generation. J. Opt. Soc. Am. B 1994, 11, 2206-2215.

(2) Frisch G. W.; Schlegel, H. B.; Scuseria, G. E.; Robb, M. A.; Cheeseman, J. R.; Scalmani, G.; Barone, V.; Petersson, G. A.; Nakatsuji, H.; Li, X., et al. Gaussian 16, Rev. A.03. Gaussian, Inc., Wallingford, CT 2016.

(3) Fujiyoshi, S.; Ishibashi, T.; Onishi, H. Low-Frequency Vibrations of Molecular Submonolayers Detected by Time-Domain Raman Spectroscopy. J. Mol. Struct. 2005, 735-736, 169-177.

(4) Lee, Y.; Das, S.; Malamakal, R. M.; Meloni, S.; Chenoweth, D. M.; Anna, J. M. Ultrafast Solvation Dynamics and Vibrational Coherences of Halogenated Boron-Dipyrromethene Derivatives Revealed through Two-Dimensional Electronic Spectroscopy. J. Am. Chem. Soc. 2017, 139, 14733-14742.

(5) Fleming, G. R.; Cho, M. Chromophore-Solvent Dynamics. Annu. Rev. Phys. Chem. 1996, 47, 109-143.

(6) McHale, J. L. Subpicosecond Solvent Dynamics in Charge-Transfer Transitions: Challenges and Opportunities in Resonance Raman Spectroscopy. Acc. Chem. Res. 2001, 34, 265-272.

(7) Joo, T.; Jia, Y.; Yu, J. Y.; Lang, M. J.; Fleming, G. R. Third-Order Nonlinear Time Domain Probes of Solvation Dynamics. J. Chem. Phys. 1996, 104, 6089-6108.

(8) Horng, M. L.; Gardecki, J. A.; Papazyan, A.; Maroncelli, M. Subpicosecond Measurements of Polar Solvation Dynamics: Coumarin 153 Revisited. J. Phys. Chem. 1995, 99, 17311-17337.

(9) Caram, J. R.; Fidler, A. F.; Engel, G. S. Excited and Ground State Vibrational Dynamics Revealed by Two-Dimensional Electronic Spectroscopy. J. Chem. Phys. 2012, 137, 024507.

(10) Butkus, V.; Zigmantas, D.; Valkunas, L.; Abramavicius, D. Vibrational vs. Electronic Coherences in 2D Spectrum of Molecular Systems. Chem. Phys. Lett. 2012, 545, 40-43.

(11) Turner, D. B.; Dinshaw, R.; Lee, K. K.; Belsley, M. S.; Wilk, K. E.; Curmi, P. M. G.; Scholes, G. D. Quantitative Investigations of Quantum Coherence for a Light-Harvesting Protein at Conditions Simulating Photosynthesis. Phys. Chem. Chem. Phys. 2012, 14, 4857-4874.

(12) Egorova, D. Oscillations in Two-Dimensional Photon-Echo Signals of Excitonic and Vibronic Systems: Stick-Spectrum Analysis and Its Computational Verification. J. Chem. Phys. 2014, 140, 034314.

(13) Egorova, D. Self-Analysis of Coherent Oscillations in Time-Resolved Optical Signals. $J$. Phys. Chem. A 2014, 118, 10259-10267.

(14) Hamm, P.; Zanni, M. Concepts and Methods of 2D Infrared Spectroscopy; Cambridge University Press: Cambridge, 2011.

(15) Mukamel, S. Principles of Nonlinear Optical Spectroscopy; Oxford University Press: New York, 1999.

(16) Gu, Y.; Widom, A.; Champion, P. M. Spectral Line Shapes of Damped Quantum Oscillators: Applications to Biomolecules. J. Chem. Phys. 1994, 100, 2547-2560.

(17) Stratt, R. M.; Maroncelli, M. Nonreactive Dynamics in Solution: The Emerging Molecular View of Solvation Dynamics and Vibrational Relaxation. J. Phys. Chem. 1996, 100, 12981-12996. 
(18) Maroncelli, M. The Dynamics of Solvation in Polar Liquids. J. Mol. Liq. 1993, 57, 1-37.

(19) Lee, Y.; Gorka, M.; Golbeck, J. H.; Anna, J. M. Ultrafast Energy Transfer Involving the Red Chlorophylls of Cyanobacterial Photosystem I Probed through Two-Dimensional Electronic Spectroscopy. J. Am. Chem. Soc. 2018, 140, 11631-11638.

(20) Lee, Y.; Malamakal, R. M.; Chenoweth, D. M.; Anna, J. M. Halogen Bonding Facilitates Intersystem Crossing in Iodo-BODIPY Chromophores. J. Phys. Chem. Lett. 2020, 11, 877-884. 